\title{
Renormalization of the Deuteron with One Pion Exchange
}

\author{
M. Pavón Valderrama ${ }^{1, *}$ and E. Ruiz Arriola, ${ }^{1,0}$ \\ ${ }^{1}$ Departamento de Física Moderna, Universidad de Granada, E-18071 Granada, Spain.
}

(Dated: November 6, 2018)

\begin{abstract}
We analyze the deuteron bound state through the One Pion Exchange Potential. We pay attention to the short distance peculiar singularity structure of the bound state wave functions in coordinate space and the elimination of short distance ambiguities by selecting the regular solution at the origin. We determine the so far elusive amplitude of the converging exponential solutions at the origin. All bound state deuteron properties can then be uniquely deduced from the deuteron binding energy, the pion-nucleon coupling constant and pion mass. This generates correlations among deuteron properties. Scattering phase shifts and low energy parameters in the ${ }^{3} S_{1}-{ }^{3} D_{1}$ channel are constructed by requiring orthogonality of the positive energy states to the deuteron bound state, yielding an energy independent combination of boundary conditions. We also analyze from the viewpoint of short distance boundary conditions the weak binding regime on the light of long distance perturbation theory and discuss the approach to the chiral limit.
\end{abstract}

PACS numbers: 03.65.Nk,11.10.Gh,13.75.Cs,21.30.Fe,21.45.+v

Keywords: NN-interaction,Renormalization,One Pion Exchange, Deuteron, NN Scattering

\section{INTRODUCTION}

Pion dynamics plays a dominant role in the low energy structure of the Nucleon-Nucleon interaction, and in particular in the description of light nuclei like the deuteron [1]. The long distance part of the interaction is given by one, two and higher pion exchanges and the fact that the deuteron is a weakly bound state suggests that many of its properties can indeed be explained in terms of these dynamical degrees of freedom in a model independent way and regardless on the less known short distance interaction. Glendenning and Kramer [2] in the early sixties recognized clear correlations between several deuteron observables generated by truncating the One Pion Exchange (OPE) potential at a distance $R=0.4915 \mathrm{fm}$ and assuming a hard core inside. Tight constraints on deuteron observables were established by Klarsfeld, Martorell and Sprung 3, 4] by integrating the deuteron wave function from infinity down to a cut-off radius using the OPE potential and rigorous inequalities. An accurate determination of the $\mathrm{D} / \mathrm{S}$ asymptotic ratio was made by Ericson and Rosa-Clot 5, 6] based on assuming the OPE correlation between the S and D wave functions and taking realistic potential models to describe the $\mathrm{S}$ wave function. (for a review on these developments see e.g Ref. [7]). Friar et al. use a multipole form factor [8] whereas Ballot et al. used separate monopole forms factor for the central and tensor part of the OPE potential mimicking the finite size of nucleons [9, 10]. Along a similar line of investigation Sprung et al. used a square well potential 11] for the central component and a vanishing potential for the tensor component.

\footnotetext{
*Electronic address: mpavon@ugr.es
}

${ }^{\dagger}$ Electronic address: earriola@ugr.es
Within the effective field theory (EFT) approach to nuclear physics proposed by Weinberg [12] the situation was revisited from a somewhat different perspective since the OPE potential appears as the lowest order of a perturbative hierarchy based on chiral symmetry [13] (for a review see e.g. Ref. [14]), and short distance ambiguities could be eliminated by the renormalization program if the auxiliary regulator is removed from the theory at the end of the calculation. This cut-off independence should occur at any level of approximation, no matter how many pions are exchanged. At long distances, renormalization group methods suggest that one is close to an infrared fixed point [15]. The renormalization procedure can be explicitly and analytically carried out within perturbation theory 16]. However, these nice features become a non trivial numerical problem beyond perturbation theory motivating the use of truncation cut-off schemes. The work of Ref. [17] uses a gaussian cut-off in coordinate space to regulate the contact delta interaction, Ref. [18] proposes the use of a subtraction method in momentum space regulating the central part, Ref. [19] uses a sharp momentum cut-off and in Ref. 20] it was proposed to use a finite short distance cut-off, whereas Ref. 21] puts exponentially suppressed regulators in momentum space. It should be mentioned that in all cases the corresponding coordinate/momentum space cut-off parameter $a / \Lambda$ is uncomfortably large/small from the viewpoint of renormalization theory. Typically, one has $a \sim 1.4 \mathrm{fm}$ (see e.g. Ref. [20]) and $\Lambda=600 \mathrm{MeV}$ 17] respectively. So, it is not obvious that according to the basic principles of EFT the short distance ambiguities are, as one might expect, indeed under control. Moreover, the existence of a well behaved finite renormalized limit is never guaranteed $a$ priori and one relies mainly on numerics. Actually, the fact that the results on deuteron observables look rather similar, regardless on the particular way how the potential is modeled at short distances, proves that the long distance pion dynamics dominates the physics confirming 
the findings of Glendenning and Kramer [2] more than 40 years ago, but does not resolve the mathematical problem whether the OPE potential can make unambiguous predictions regardless of any short distance physical scale.

The OPE potential is local in coordinate space where the problem is naturally formulated by the standard Schrödinger framework. Moreover, it is singular at the origin and giving boundary conditions at that point is not a well defined procedure for uniquely determining the solution 22] (for a comprehensive review in the one channel case see e.g. Ref. 23]). There is the added difficulty that we have two coupled second order differential equations. In the deuteron channel one has four independent solutions, which according to their singularity structure correspond to either two regular and two irregular solutions at infinity or three regular and one irregular solution at the origin. The normalizability condition of the deuteron wave functions eliminates all constants for a given deuteron binding energy, which instead of being predicted has to be treated as an independent parameter. One of the advantages of the coordinate space treatment of renormalization is that it can directly be extended to other singular cases such as the Two Pion Exchange (TPE) potential 24] which is also finite everywhere except the origin. In contrast, momentum space treatments require an extra regularization of the potential besides the standard cut-off regularization of the Lippmann-Schwinger equation.

The authors of Ref. 11] found a discrete sequence of equivalent short distance cut-off radii having almost the same deuteron properties. In their analysis of the problem one regular solution at the origin with a converging exponential behavior, $\exp \left(-4(2 R / r)^{\frac{1}{2}}\right)$ with $R \sim 1 \mathrm{fm}$, was discarded on numerical grounds. The same result was also implicitly used in Ref. 25] and large $N_{c}$ arguments in favor of it were raised in Ref. [26]. This extra condition would actually predict the deuteron binding energy from the OPE potential. As we will show in this paper, the converging exponential is non-vanishing although rather elusive because its contribution to the deuteron wave function only becomes sizeable at relatively large distances and accurate numerical work must be done to pin down its value with a certain degree of confidence.

In the present work we show that there is no need to truncate the OPE potential on a physical scale to produce unique and cut-off independent predictions for deuteron properties and scattering observables in terms of the OPE potential parameters and the deuteron binding energy. These might then legitimately be called OPE model independent predictions and paves the way for a systematic investigation on the case where more pions are exchanged and other effects are taken into account 24]. After presenting the basic notation in Sect. $1 \mathrm{~A}$ we discuss in Sect. IIB the regular solutions at the origin and establish that the limit when the regulator is removed is finite. For numerical purposes it is useful to define some short distance regulator as an auxiliary tool. In Sect. IIC we use six different regulators based on boundary conditions of the wave function and check for stability to high precision for all regulators. This procedure generates correlations among deuteron observables if the deuteron binding energy is varied as a free and independent parameter as we do in Sect. IIII A particularly interesting situation is provided by the weak binding limit, which can be taken with fixed OPE potential parameters. In such a case the long distance behavior should dominate and one might expect perturbative methods to apply and be compared to the exact OPE calculation. The details of the perturbative calculation are postponed to Appendix $₫$ where we present a coordinate space version of the method, in consonance with the exact treatment. A detailed comparison shows that the perturbative argument is too naive and would only hold in the weak coupling regime as well, due to the appearance of non-analytical contributions in the $\pi N N$ coupling constant. Mathematically, we show that it is not possible to go beyond first order since the coefficients of the expansion diverge. Numerically, the disagreement at first order is typically on the $30 \%$ level for physical values of the OPE parameters at zero binding. After Ref. [27] the chiral limit in nuclear physics has attracted considerable attention in recent works [28, 29, 30] and also the limit of heavy pions in connection with lattice QCD calculations, where the pion mass is still far from its physical value. We study in Sect. IV the correlations among those observables if the pion mass is varied away from its physical value by studying a suitable extension of the Feynman-Helmann theorem. Another remarkable property of the OPE potential which we deal with in Sect. $\nabla$ is that low energy parameters as well as the scattering phase-shifts can be uniquely determined from the OPE potential parameters and the deuteron binding, due to orthogonality constraints of the bound state and scattering states. In Sect. VI the determination of the non-vanishing coefficient of the converging exponential at the origin is carried out by a short distance expansion to eighth order of the OPE deuteron wave functions. In Sect. VII we come to the conclusions.

One of the surprising results in the OPE description of the deuteron has to do with the small asymptotic ratio between the $\mathrm{D}$ and $\mathrm{S}$ waves, $w(\infty) / u(\infty)=\eta=0.0256$ coming from a large ratio at short distances of order unity, $w(0) / u(0)=1 / \sqrt{2}=0.707$. Although this feature is specific to the OPE potential it is somewhat a bit outside the main topic of this work. So we relegate this issue to Appendix $[$ where we show how this can be easily understood if a local rotation of the deuteron wave functions diagonalizing the coupled channel potential is carried out. Obviously, such a transformation cannot simultaneously diagonalize the kinetic terms, but the residual mixing is related to the derivative of a local mixing angle which numerically turns out to be a slowly varying function. Using this as a starting approximation we can determine in a perturbative fashion the asymptotic D/S ratio yielding the exact OPE value with a $1 \%$ accuracy. 


\section{BOUND STATE EQUATIONS AND THEIR SOLUTIONS}

\section{A. The OPE deuteron Equations}

The Deuteron coupled channel ${ }^{3} S_{1}-{ }^{3} D_{1}$ set of equations read

$$
\begin{aligned}
-u^{\prime \prime}(r)+U_{s}(r) u(r)+U_{s d}(r) w(r) & =-\gamma^{2} u(r), \\
-w^{\prime \prime}(r)+U_{s d}(r) u(r)+\left[U_{d}(r)+\frac{6}{r^{2}}\right] w(r) & =-\gamma^{2} w(r),
\end{aligned}
$$

together with the asymptotic conditions at infinity

$$
\begin{aligned}
u(r) & \rightarrow A_{S} e^{-\gamma r}, \\
w(r) & \rightarrow A_{D} e^{-\gamma r}\left(1+\frac{3}{\gamma r}+\frac{3}{(\gamma r)^{2}}\right),
\end{aligned}
$$

where $\gamma=\sqrt{M B}$ is the deuteron wave number, $A_{S}$ is the normalization factor and the asymptotic $\mathrm{D} / \mathrm{S}$ ratio parameter is defined by $\eta=A_{D} / A_{S}$. The ${ }^{3} S_{1}-{ }^{3} D_{1}$ coupled channel potential is given by

$$
U_{s}=U_{c}, \quad U_{s d}=2 \sqrt{2} U_{T}, \quad U_{d}=U_{C}-2 U_{T},
$$

where the OPE reduced potential $(U=2 \mu V)$ is given for $r>0$ by

$$
\begin{aligned}
U_{C} & =-\frac{m M g_{A}^{2}}{16 \pi f_{\pi}^{2}} \frac{e^{-m r}}{r} \\
U_{T} & =-\frac{m^{2} M g_{A}^{2}}{16 \pi f_{\pi}^{2}} \frac{e^{-m r}}{r}\left(1+\frac{3}{m r}+\frac{3}{(m r)^{2}}\right),
\end{aligned}
$$

where $m$ is the pion mass, $M=2 \mu_{n p}=2 M_{n} M_{p} /\left(M_{n}+\right.$ $M_{p}$ ) twice the reduced proton-neutron mass, $g_{A}$ the axial nucleon coupling constant and $f_{\pi}$ the pion weak decay constant. Note that we assume this potential to be valid for any strictly positive distance, $r \neq 0$, so the limit $r \rightarrow 0^{+}$will be carefully taken, without subtracting any contribution in the potential.

It is convenient to define the length scale

$$
R=\frac{3 g_{A}^{2} M}{32 \pi f_{\pi}^{2}}
$$

which value is around $1 \mathrm{fm}$. For numerical calculations we take $f_{\pi}=92.4 \mathrm{MeV}, M=938.918 \mathrm{MeV}, g_{A}=1.29$ and hence $R=1.07764 \mathrm{fm}$. Using the Goldberger-Treiman relation, $g_{\pi N N}=g_{A} M / f_{\pi}$, the corresponding pion nucleon coupling constant is $g_{\pi N N}=13.1083$ according to a phase shift analysis of NN scattering [31]. Nevertheless, after the latest determinations from the GMO sum rule 32 we will also take the value $g_{\pi N N}=13.3158$. As we will see this variation at the $5 \%$ level dominates the uncertainties in the OPE calculations.

\section{B. The short distance regular solutions}

We look for normalized functions of the Eqs. (2),

$$
1=\int_{0}^{\infty}\left(u(r)^{2}+w(r)^{2}\right) d r,
$$

from which $A_{S}$ can be determined. The normalization at infinity is guaranteed due to the asymptotic conditions, Eq. (3). However, the coupled channel potential becomes singular at short distances, since $U_{T} \rightarrow-2 R / r^{3}$. Keeping only this term in Eqs. (2) one can decouple the equations through the unitary transformation [1]

$$
\begin{aligned}
& u_{A}(r)=\sqrt{\frac{2}{3}} u(r)+\frac{1}{\sqrt{3}} w(r), \\
& u_{R}(r)=-\frac{1}{\sqrt{3}} u(r)+\sqrt{\frac{2}{3}} w(r),
\end{aligned}
$$

yielding an attractive singular potential $U_{A} \rightarrow-4 R / r^{3}$ for $u_{A}$ and $U_{R} \rightarrow 8 R / r^{3}$ for $u_{R}$. Any solution obtained by integrating from infinity with the Eq. (3) down to the origin has the asymptotic short distance behavior ${ }^{1}$,

$$
\begin{aligned}
& u_{R}(r) \rightarrow\left(\frac{r}{R}\right)^{3 / 4}\left[C_{1 R} e^{+4 \sqrt{2} \sqrt{\frac{R}{r}}}+C_{2 R} e^{-4 \sqrt{2} \sqrt{\frac{R}{r}}}\right], \\
& u_{A}(r) \rightarrow\left(\frac{r}{R}\right)^{3 / 4}\left[C_{1 A} e^{-4 i \sqrt{\frac{R}{r}}}+C_{2 A} e^{4 i \sqrt{\frac{R}{r}}}\right] .
\end{aligned}
$$

The constants $C_{1 R}, C_{2 R}, C_{1 A}$ and $C_{2 A}$ depend on both $\gamma$ and $\eta$ and the OPE potential parameters, $g_{\pi N N}$ and $m$. Note that the leading short distance $r$ dependence does not involve the pion mass and the deuteron wave number. Higher order corrections to these solutions can be computed systematically to high orders and are presented below in Sect. VI

The regular solution at infinity contains the normalization constant $A_{S}$, which is customarily set to one for computational purposes, the deuteron wave number $\gamma$ and the asymptotic D/S ratio parameter $\eta$. The normalizability of the wave function at the origin requires

$$
C_{1 R}(\gamma, \eta)=0
$$

which is a relation between $\eta$ and $\gamma$. The other remaining constants are then completely fixed. This means that for the OPE potential, the deuteron binding energy can be used as an independent parameter. Thus, one has three independent variables, $\gamma$, the coupling constant with length scale dimension $R$ (or equivalently $g_{\pi N N}$ ) and the pion mass $m$. Obviously, this suggests integrating in from infinity and determining $\eta$ from the regularity condition at the origin (11).

\footnotetext{
1 The solutions for $u_{A}$ and $u_{R}$ are written in terms of spherical Bessel functions [11]. We keep the leading short distance behavior only.
} 
To analyze whether some additional condition arises let us check the selfadjointness of the coupled channel Hamiltonian. The flux at a point $r$ is given by

$$
\begin{aligned}
i J(r) & =u^{*}(r)^{\prime} u(r)-u^{*}(r) u^{\prime}(r) \\
& +w^{*}(r)^{\prime} w(r)-w^{*}(r) w^{\prime}(r),
\end{aligned}
$$

so that current probability conservation at the origin implies

$$
\left|C_{1 A}\right|^{2}-\left|C_{2 A}\right|^{2}=2 \sqrt{2} i\left(C_{1 R}^{*} C_{2 R}-C_{2 R}^{*} C_{1 R}\right) .
$$

Thus, if we set $C_{1 R}=0$ there is no condition on $C_{2 R}$ and one has $C_{1 A}=C_{A} e^{i \varphi}$ and $C_{2 A}=C_{A} e^{-i \varphi}$ with $C_{A}$ and $\varphi$ real. So, we have three constants, $C_{2 R}(\gamma), C_{A}(\gamma)$ and $\varphi(\gamma)$, characterizing the normalizable solutions at short distances for a given value of the deuteron wave number $\gamma$,

$$
\begin{aligned}
& u_{R}(r) \rightarrow C_{R}(\gamma)\left(\frac{r}{R}\right)^{3 / 4} e^{-4 \sqrt{2} \sqrt{\frac{R}{r}}} \\
& u_{A}(r) \rightarrow C_{A}(\gamma)\left(\frac{r}{R}\right)^{3 / 4} \sin \left[4 \sqrt{\frac{R}{r}}+\varphi(\gamma)\right] .
\end{aligned}
$$

Actually, if we have any other state, say a scattering state with positive energy, unitarity (i.e. orthogonality) requires that the constant $\varphi(k)$ coincides with the bound state phase $\varphi(\gamma)$. We will come back to this issue later when discussing low energy parameters and scattering solutions in Sect. $\nabla$ It is natural to expect that some combination of short distance constants is independent on the OPE potential parameters as they encode short distance physics. In Sect. IV we establish, by demanding the standard Feynman-Hellmann theorem, that specifically the short distance phase $\varphi$ does not depend on the OPE potential parameters. In Sect. $\nabla 1$ we determine the values of the three constants characterizing the three regular solutions by a detailed short distance analysis of the OPE deuteron wave functions.

Note that any additional condition would actually predict both $\gamma$ and $\eta$ from $m$ and $R$. This contradicts the claim of Ref. 11] that $C_{2 R}=0$, a conclusion implicitly used in Ref 25] and supported by the large $N_{c}$ argument of Ref. [26]. On the other hand, if one takes the experimental values of $\eta$ and $\gamma$ as done in Ref. 20] one obtains both non vanishing $C_{1 R}$ and $C_{2 R}$ i.e., the irregular non-normalizable solution, unless a short distance cut-off, $R>0.8 \mathrm{fm}$, is introduced as a physical scale and not as an auxiliary removable regulator.

\section{Regularization with boundary conditions}

Ideally, one would integrate in the large asymptotic solutions, Eq. (3), and match the short distance behavior of Eq. (11) imposing the regularity condition (11). In practice, however, the converging exponential at the origin is rather elusive since integrated-in solutions quickly run into the diverging exponentials due to round-off errors for $r \sim 0.05 \mathrm{fm}$ and dominate over the converging exponential. The reason has to do with the fact that the natural scale where both exponentials are comparable is rather large $r=4 \sqrt{2} R \sim 6 \mathrm{fm}$, but in that region the lowest order short distance approximation does not hold.

Instead, we will also try putting several short distance boundary conditions corresponding to the choice of regular solutions at the origin,

$$
\begin{aligned}
& u(a)=0 \quad(\mathrm{BC} 1), \\
& u^{\prime}(a)=0 \quad(\mathrm{BC} 2), \\
& w(a)=0 \quad \text { (BC3), } \\
& w^{\prime}(a)=0 \quad \text { (BC4), } \\
& u(a)-\sqrt{2} w(a)=0 \quad(\mathrm{BC} 5), \\
& u^{\prime}(a)-\sqrt{2} w^{\prime}(a)=0 \quad(\mathrm{BC} 6),
\end{aligned}
$$

The advantage of using this kind of short distance cutoffs based on a boundary condition is that there is only a single scale in the problem as one naturally expects, and that one never needs to declare what is the wave function below the boundary radius. Putting a square well potential as a counter-term [25] with depth $U_{0}$ appears natural from standard perturbative experience but needs specification of a further length scale, $1 / \sqrt{U_{0}}$, and moreover, generates multi-valuation ambiguities [34, 35].

It is convenient to use the superposition principle of boundary conditions to write

$$
\begin{gathered}
u(r)=u_{S}(r)+\eta u_{D}(r) \\
w(r)=w_{S}(r)+\eta w_{D}(r),
\end{gathered}
$$

where $\left(u_{S}, w_{S}\right)$ and $\left(u_{D}, w_{D}\right)$ correspond to the boundary conditions at infinity, Eq. (3) with $A_{S}=1$ and $A_{D}=0$ and with $A_{S}=0$ and $A_{D}=1$ respectively. Thus, at the boundary we can impose any of the conditions by just eliminating $\eta^{2}$. The resulting $\eta$ value obtained by all these boundary conditions is presented in Fig. 1. Actually, we see that the boundary condition $u^{\prime}(a)-\sqrt{2} w^{\prime}(a)$ is about the smoothest condition we can think of, since the $u_{R}$ combination goes to zero at small distances, its derivative, $u_{R}^{\prime}$ also goes to zero, although a bit less faster since $u_{R}^{\prime} / u_{R} \sim 1 / r^{3 / 2}$. We see that all determinations of $\eta$ based on any of the proposed cut-offs yield the same value with great accuracy at cut-off radii below $0.2 \mathrm{fm}$.

\footnotetext{
${ }^{2}$ Numerically we find at the cut-off boundary $r=0.2 \mathrm{fm}$

$$
\begin{aligned}
u(0.2) & =1139.23-43263.2 \eta \\
w(0.2) & =-1807.33+68632.5 \eta \\
u^{\prime}(0.2) & =-35529.8+1.34913 \cdot 10^{6} \eta
\end{aligned}
$$$$
w^{\prime}(0.2)=55194.3-2.09606 \cdot 10^{6} \eta
$$

These large numbers appear because the of the dominance of the diverging exponential at short distances. 


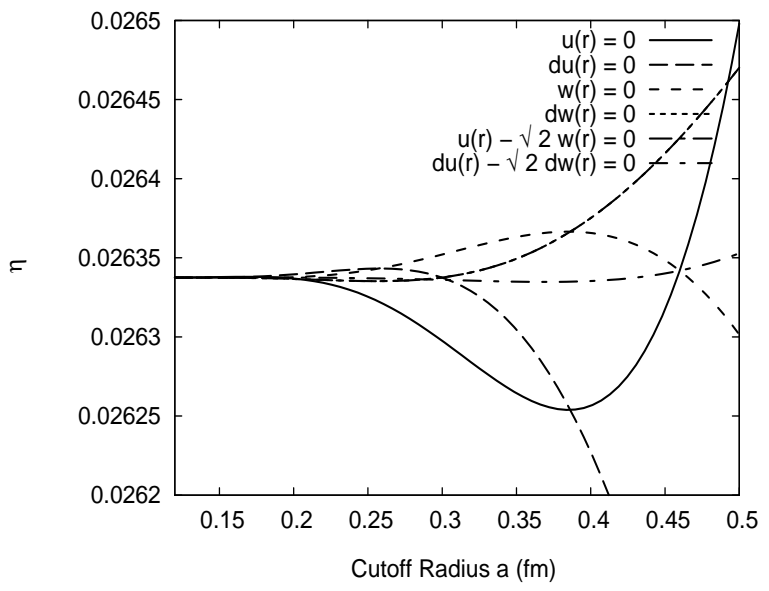

FIG. 1: The dependence of the asymptotic $D / S$ ratio $\eta$ on the cut-off radius $a$ for several boundary conditions. We use $m=$ $138.03 \mathrm{MeV}$ and $R=1.07764 \mathrm{fm}$ (corresponding to $g_{\pi N N}=$ $13.1083)$.

This is somewhat fortunate since arithmetic precision is outraged typically for $r<0.06 \mathrm{fm}$. Obviously, any short distance cut-off generates finite cut-off effects in the wave functions for distances close to the cut-off radius. In Sect. VI we analyze this problem by matching solutions of the form of Eq. (14) to the integrated in numerical solutions and find that for many practical purposes these finite cut-off effects are negligible. Thus, we will base most of our results on the "smoothest" condition BC6 of Eq. (15).

The resulting deuteron wave functions $u$ and $w$ obtained by integrating in from infinity to the origin the OPE potential are plotted in Fig. 2] where the irregular solutions are obtained with the experimental $D / S$ ratio $\eta_{d}=0.0256$ and the regular ones with the OPE $D / S$ ratio $\eta_{\mathrm{OPE}}=0.026333$. For comparison we also plot the NijmII deuteron wave functions. We emphasize that the value of $\eta$ is a direct consequence of taking the OPE down to the origin seriously.

\section{Deuteron observables}

Once the solutions are known we can determine several observables of interest. The matter radius reads,

$$
r_{m}^{2}=\frac{1}{4}\left\langle r^{2}\right\rangle=\frac{1}{4} \int_{0}^{\infty} r^{2}\left(u(r)^{2}+w(r)^{2}\right) d r
$$

while potential contribution to the quadrupole moment (without meson exchange currents)

$$
Q_{d}=\frac{1}{20} \int_{0}^{\infty} r^{2} w(r)(2 \sqrt{2} u(r)-w(r)) d r
$$

An important observable is the deuteron inverse radius

$$
\left\langle r^{-1}\right\rangle=\int_{0}^{\infty} d r \frac{u(r)^{2}+w(r)^{2}}{r}
$$

which appears in low energy pion-deuteron scattering. Finally, the $D$-state probability is given by

$$
P_{D}=\int_{0}^{\infty} w(r)^{2} d r
$$

Both $P_{D}$ and $\left\langle r^{-1}\right\rangle$ are sensitive to the intermediate distance region around $2 \mathrm{fm}$ whereas $Q_{d}$ and $r_{m}$ get their contribution from larger distances $\sim 4 \mathrm{fm}$.

The results for the asymptotic S-wave normalization $A_{S}$, the matter radius $r_{m}$, the quadrupole moment, $Q_{d}$, and the D-state probability, $P_{D}$ are presented in Table [ The errors in the numerical calculation have been assessed by varying the short distance cut-off in the range $a=0.1-0.2 \mathrm{fm}$ (in momentum space that would naively correspond to take $\Lambda=1 / a=2-4 \mathrm{GeV})$. As we see, the cut-off uncertainty is smaller than the one induced by variations at the $2 \%$ level in the $g_{\pi N N}$ coupling constant in the range between the lowest value $(\sim 13.1)$ obtained by a fit to NN phase-shifts 31] and the highest recent value $(\sim 13.3)$ determined from the GMO sum rule [32]. Equivalently, this uncertainty corresponds to take $R=1.0776 \mathrm{fm}$ and $R=1.1108 \mathrm{fm}$ respectively. Our results are generally speaking in agreement with previous determinations where different sorts of cut-off methods have also been implemented.

\section{E. Discussion}

At this point it may prove useful to ponder on the previous results from a wider perspective. Let us remind that the basic assumption of an EFT is that the study of long wave length phenomena such as low energy scattering or weakly bound systems do not require a detailed knowledge of short distance physics. This general and widely accepted principle requires some qualification because attractive and repulsive singular potentials behave quite differently in this respect. Singular attractive potentials, $\sim 1 / r^{n}$, generate wave functions vanishing as a power law, $r^{n / 4} \sin \left(r^{-n / 2+1}+\varphi\right)$, and which need a mixed boundary condition to specify the short distance phase $\varphi$. Thus, short distance details become less important, regardless on the value of $\varphi$. On the contrary, for singular repulsive potentials the wave functions behave as $r^{n / 4} e^{ \pm r^{-n / 2+1}}$ and only for the regular solution short distance details become irrelevant. In the OPE potential, it is precisely the repulsive short distance OPE component which requires a fine tuning of the solutions and eliminates one a priori independent parameter like, e.g., the asymptotic $\mathrm{D} / \mathrm{S}$ ratio $\eta$. As we see, if $\eta$ is treated as an independent variable the short distance behavior of the deuteron wave functions precludes the definition of a normalizable state due to the onset of the irregular 


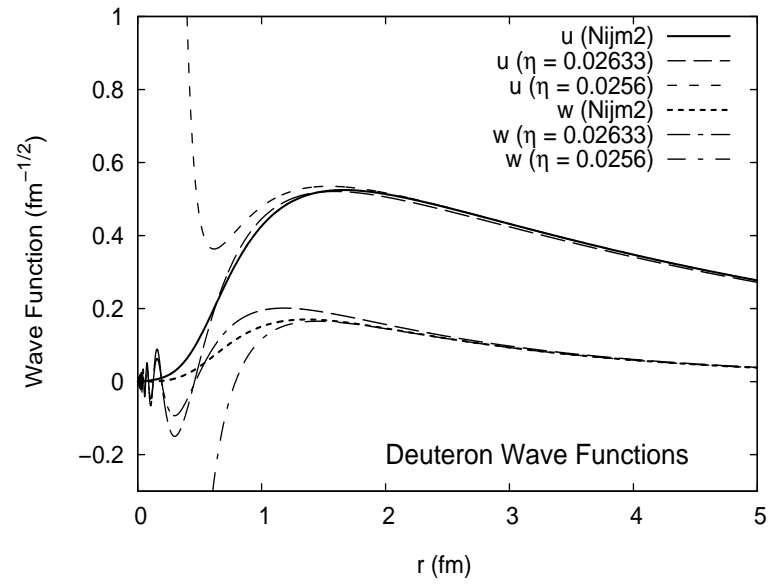

FIG. 2: The deuteron wave functions $u$ and $w$, obtained by integrating in from infinity to the origin the OPE potential, compared to those obtained with the Nijm II potential 33]. The irregular solutions are obtained with the experimental $D / S$ ratio, $\eta_{d}=0.0256$, and the regular ones with the OPE $D / S$ ratio, $\eta_{\mathrm{OPE}}=0.026333$. We use $m=138.03 \mathrm{MeV}$ and $R=1.07764 \mathrm{fm}$ (corresponding to $g_{\pi N N}=13.1083$ ).

solution. This short distance insensitivity at low energies could only be implemented by keeping the experimental $\eta$ value and ignoring OPE physics below some scale. The lower limit established in Ref. [20] to obtain a normalizable state was $a \sim 1.3 \mathrm{fm}$ for the OPE potential. This obviously requires some extension of the wave function below that scale and the pretended model independence becomes a bit obscured. Our point is that the short distance insensitivity materializes automatically for the regular OPE deuteron wave functions since they vanish at the origin.

\section{OPE CORRELATIONS IN DEUTERON OBSERVABLES}

As we have said, in the OPE potential we can use the deuteron wave number as an input of the calculation on the same footing as $g_{\pi N N}$ and the pion mass $m$. Then, other observables are predicted. We will study now the dependence of these observables on $\gamma, m$ and $R$.

\section{A. Dependence on the Binding Energy}

In Fig. 3 we show the dependence of the $\mathrm{D} / \mathrm{S}$ ratio as a function of the deuteron wave number $\gamma$ keeping $m$ and $R$ fixed. In the weak binding limit $\gamma \ll m_{\pi}$, long distances dominate and the finiteness of the wave function at a point $r \gg 1 / m$ requires $\eta \sim \gamma^{2}$. The radius of convergence of such an expansion for the observables is $|\gamma|<m / 2$, since the integrals involve the factor $e^{-(2 \gamma+m) r}$ at large distances, diverging for $\gamma<-m / 2$. The experimental number is not far from $\gamma=m / 3$, which is within the domain of analyticity but somewhat close to the convergence radius. So, one expects a slow convergence. As we see in the weak binding limit we have a quadratic behavior $\eta_{\mathrm{OPE}} \sim \gamma^{2}$ whereas for stronger binding a linear behavior sets in. It is remarkable that the experimental values in the intermediate regime. On the other hand, in the strong binding case $\gamma \gg m_{\pi}$, short distances dominate and we must have $\eta \sim 1 / \sqrt{2}$. Numerically we find for the deuteron observables,

$$
\begin{aligned}
\eta^{\mathrm{OPE}} & =0.9638 \gamma^{2}-3.46864 \gamma^{3}+\mathcal{O}\left(\gamma^{4}\right) \\
\frac{A_{S}^{\mathrm{OPE}}}{\sqrt{2 \gamma}} & =1+1.2455 \gamma-0.4705 \gamma^{2}+\mathcal{O}\left(\gamma^{3}\right) \\
\sqrt{8} \gamma r_{m}^{\mathrm{OPE}} & =1+1.2455 \gamma-0.4705 \gamma^{2}+\mathcal{O}\left(\gamma^{3}\right) \\
Q_{d}^{\mathrm{OPE}} & =0.6815-3.5437 \gamma+\mathcal{O}\left(\gamma^{2}\right)
\end{aligned}
$$

Note that we have the weak binding correlation

$$
r_{m}=\frac{A_{S}}{4 \gamma^{3 / 2}}+\mathcal{O}\left(\gamma^{3}\right)
$$

which is compatible at the $2 \sigma$ confidence level with data; for the experimental value $A_{S}=0.8845(8)$ the value $r_{m}=1.984(2)$ to be compared with the experimental number $r_{m}=1.971(6)$. In the weak binding limit one also has the correlation

$$
\frac{\sqrt{2} \gamma^{2} Q_{d}}{\eta_{d}}=1+\mathcal{O}(\gamma)
$$

a dependence that one would expect on general grounds by just taking the asymptotic formulas and neglecting the $w(r)^{2}$ term in the expression for the quadrupole moment. Experimentally this relation is fulfilled with a $15 \%$ accuracy. The non-perturbative OPE value is actually closer to potential models.

\section{B. Comparison with perturbation theory}

It is instructive to solve the coupled deuteron equations, Eq. (2) in standard perturbation theory for the fixed energy bound state. One of the reasons is to check the correctness of our non-perturbative calculations in the weak binding regime. Another motivation is to establish contact with the perturbative calculations of Ref. 16] where dimensional regularization in the power divergence subtraction (PDS) scheme was implemented. Finally, there is the question of quantitatively assessing the validity of such an approximation. We relegate the calculation to Appendix A At first order in perturbation theory one 
TABLE I: Deuteron properties for the OPE compared to the short range approximation and first order perturbation theory. We use the non-relativistic relation $\gamma=\sqrt{2 \mu_{n p} B}$ with $B=2.224575(9)$ and take $m=138.03 \mathrm{MeV}$ and $R=(3 / 8 M) g_{\pi N N}^{2} /(4 \pi)=$ $1.07764 \mathrm{fm}$ corresponding to $g_{\pi N N}=13.1083$ [31] (except the row OPE* where the value $g_{\pi N N}=13.316$ [32] has been taken). The error is estimated by changing the short distance cut-off in the range $a=0.1-0.2 \mathrm{fm}$.

\begin{tabular}{|c|c|c|c|c|c|c|c|c|c|c|c|}
\hline & $\gamma\left(\mathrm{fm}^{-1}\right)$ & $\eta$ & $A_{S}\left(\mathrm{fm}^{-1 / 2}\right)$ & $r_{m}(\mathrm{fm})$ & $Q_{d}\left(\mathrm{fm}^{2}\right)$ & $P_{D}$ & $\left\langle r^{-1}\right\rangle$ & $\alpha_{0}(\mathrm{fm})$ & $\alpha_{02}\left(\mathrm{fm}^{3}\right)$ & $\alpha_{2}\left(\mathrm{fm}^{5}\right)$ & $r_{0}(\mathrm{fm})$ \\
\hline Short & Input & 0 & 0.6806 & 1.5265 & 0 & $0 \%$ & $\infty$ & 4.3177 & 0 & 0 & 0 \\
\hline $\mathrm{OPE}$ (pert) & Input & 0.051 & 0.7373 & 1.6429 & 0.4555 & $0 \%$ & $\infty$ & 4.6089 & 2.5365 & 0 & 0.4831 \\
\hline OPE & Input & 0.02633 & $0.8681(1)$ & $1.9351(5)$ & $0.2762(1)$ & $7.88(1) \%$ & $0.476(3)$ & $5.335(1)$ & $1.673(1)$ & $6.169(1)$ & $1.638(1)$ \\
\hline $\mathrm{OPE}^{*}$ & Input & 0.02687 & $0.8718(2)$ & $1.9429(6)$ & $0.2826(2)$ & $7.42(1) \%$ & $0.471(3)$ & $5.353(1)$ & $1.715(1)$ & $6.4001(1)$ & $1.663(1)$ \\
\hline NijmII & Input & 0.02521 & $0.8845(8)$ & 1.9675 & 0.2707 & $5.635 \%$ & 0.4502 & 5.418 & 1.647 & 6.505 & 1.753 \\
\hline Reid93 & Input & 0.02514 & $0.8845(8)$ & 1.9686 & 0.2703 & $5.699 \%$ & 0.4515 & 5.422 & 1.645 & 6.453 & 1.755 \\
\hline Exp. ${ }^{a}$ & 0.231605 & $0.0256(4)$ & $0.8846(9)$ & $1.9754(9)$ & $0.2859(3)$ & $5.67(4)$ & & $5.419(7)$ & & & $1.753(8)$ \\
\hline
\end{tabular}

${ }^{a}$ (Non relativistic). See e.g. Ref. [37] and references therein.

gets in the weak binding limit

$$
\begin{aligned}
\eta_{\text {pert }} & =1.5497 \gamma^{2}-4.15479 \gamma^{3}+\mathcal{O}\left(\gamma^{4}, R^{2}\right) \\
\frac{A_{S, \text { pert }}}{\sqrt{2 \gamma}} & =1-0.7184 \gamma-2.7394 \gamma^{2}+\mathcal{O}\left(\gamma^{3}, R^{2}\right) \\
r_{m, \text { pert }} \sqrt{8} \gamma & =1+0.71843 \gamma-2.7394 \gamma^{2}+\mathcal{O}\left(\gamma^{3}, R^{2}\right) \\
Q_{\text {pert }} & =1.09587-5.87576 \gamma+\mathcal{O}\left(\gamma^{2}, R^{2}\right)
\end{aligned}
$$

The nominally $\mathcal{O}\left(R^{2}\right)$ second order contributions are in fact divergent because the leading order correction to the D-wave component $w(r)$ diverges at the origin (see Appendix $\mathrm{A}$. In general terms we find that the exact OPE results are estimated within $30 \%$ by the first order perturbative calculations of Appendix. A

\section{DEPENDENCE ON THE PION MASS AND CHIRAL LIMIT}

Recent works [27, 28, 29, 30] predict the change of the deuteron binding energy as a function of the pion mass by taking the experimental binding energy at the physical value of the pion mass and making the additional assumption short distance physics to be independent on the pion mass. While it is true that the leading short distance $r$ dependence of the deuteron wave functions are independent on the pion mass, the constants $C_{A}, C_{R}$ and $\varphi$ do in principle depend on the three independent parameters $m, g_{\pi N N}$ and $\gamma$. As we have noted $\gamma$ cannot be predicted for the OPE potential. So the approach pursued in Refs. 27, 28, 29, 230] is equivalent to integrate in with the physical pion mass and then integrate out fixing some combination of short distance constants with the unphysical pion mass and searching for the appropriate regular solution at infinity. If one makes the pion lighter long distance effects should dominate, and one could just use the OPE potential to estimate the chiral limit as a first approximation. It is thus interesting to analyze the pion mass dependence both explicitly (i.e. varying $m$ in the OPE potential) and implicitly (i.e. taking into account the dependence of the OPE coupling $R$ on the pions mass). We will determine the pertinent combination of short distance constants by demanding the Feynman-Hellmann theorem in the OPE potential.

\section{A. Explicit pion mass dependence}

To proceed, let us assume that to an infinitesimal change in $m \rightarrow m+\Delta m$ there corresponds a change both in the deuteron wave number $\gamma \rightarrow \gamma+\Delta \gamma$ and in the coupled channel potential matrix $U(r) \rightarrow U(r)+\Delta U(r)$. We can write a Lagrange identity by varying the equation and its adjoint, yielding for a normalized state

$$
\begin{aligned}
-\frac{\partial \gamma^{2}}{\partial m} & =\left\langle\Psi_{m}\left|\frac{\partial U}{\partial m}\right| \Psi_{m}\right\rangle \\
& +\left.\left[u^{\prime} \frac{\partial u}{\partial m}-u \frac{\partial u^{\prime}}{\partial m}+w^{\prime} \frac{\partial w}{\partial m}-w \frac{\partial w^{\prime}}{\partial m}\right]\right|_{0} ^{\infty}
\end{aligned}
$$

This is an extended Feynman-Hellmann theorem where the second term in the l.h.s. corresponds to the short distance contribution (the term at infinity vanishes for a bound state). One of the advantages of the FeynmanHellmann theorem is that one could in principle establish comparison theorems, provided the change in the coupled channel potential matrix, $\Delta U$, is a definite quadratic form. Note also that the derivative with respect to $m$ annihilates the centrifugal term, $6 / r^{2}$, and one can diagonalize the coupled channel potential by the unitary transformation Eq. (9) so that the result behaves additively in the attractive and repulsive eigenchannels. Using the leading short distance behavior, Eq. (14), we therefore get

$$
\begin{aligned}
-\frac{\partial \gamma^{2}}{\partial m} & =\int_{0}^{\infty} d r\left[u_{A}(r)^{2} \frac{\partial U_{A}}{\partial m}+u_{R}(r)^{2} \frac{\partial U_{R}}{\partial m}\right] \\
& +C_{A}^{2} \frac{d \varphi}{d m}
\end{aligned}
$$



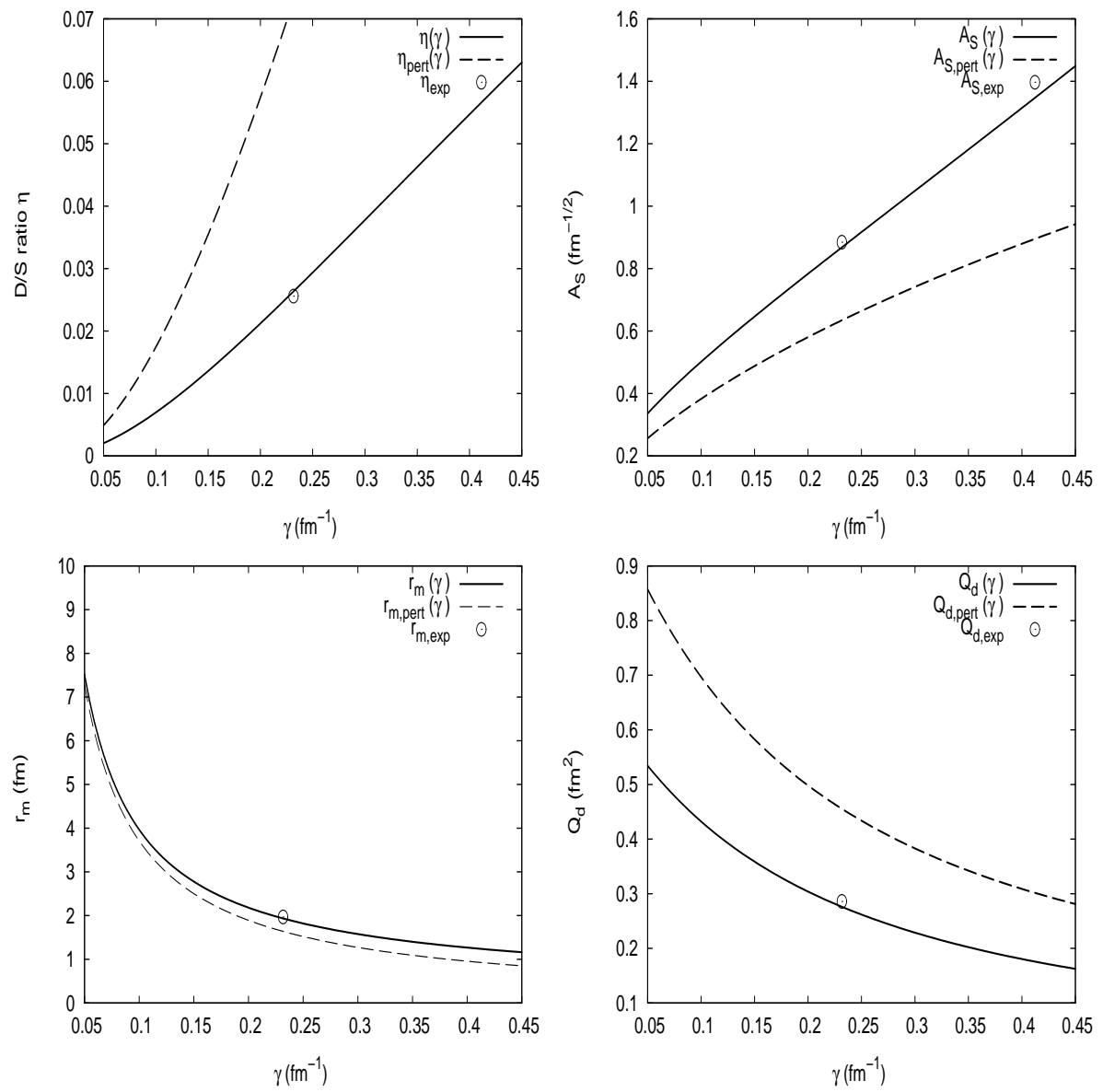

FIG. 3: The dependence of the asymptotic $D / S$ ratio $\eta$ (upper left panel), the S-wave normalization $A_{S}$ (in fm ${ }^{-1 / 2}$, upper right panel), the matter radius $r_{m}$ (in fm, lower left panel) and the quadrupole moment $Q_{d}$ (in fm ${ }^{2}$, lower right panel) on the deuteron wave number $\gamma\left(\right.$ in $\mathrm{fm}^{-1}$ ) for the short range theory, the first order perturbative result and the exact OPE result. The points represent the experimental values. We use $m=138.03 \mathrm{MeV}$ and $R=1.07764 \mathrm{fm}$.

As we see, assuming as suggested in Ref. [27] that the short distance physics does not depend on the pion mass corresponds to demanding the standard FeynmanHellmann theorem where only the OPE potential change contributes. For $C_{A} \neq 0$ one obtains the condition

$$
\frac{d}{d m} \varphi(\gamma, m)=\frac{\partial \varphi}{\partial \gamma} \frac{d \gamma}{d m}+\frac{\partial \varphi}{\partial m}=0
$$

whence a functional relation between the pion mass and the deuteron binding energy follows. However, note that even in this case the sign of the result is indefinite since

$$
\frac{\partial \gamma^{2}}{\partial m}=\int_{0}^{\infty} d r\left[u_{A}(r)^{2} \frac{\partial U_{A}}{\partial m}+u_{R}(r)^{2} \frac{\partial U_{R}}{\partial m}\right] .
$$

So, we have to determine the sign numerically.

The relation (31) has an equivalent formulation in the boundary condition regularization. For instance, if we assume the same condition BC6 of Eq. (43) for all values of the pion mass we get

$$
\frac{d}{d m}\left[\frac{w^{\prime}(a)}{u(a) \sqrt{2}+w(a)}\right]=0
$$

where $a$ is taken to be independent of $m$. In practice, one computes the ratio within the bracket for the physical pion mass and searches for $\gamma$ such that the ratio for the unphysical pion mass yields the same numerical value. If we take the chiral limit we get $B_{d}\left(0, g_{\pi N N}\right)=4.3539 \mathrm{MeV}$ This value is very close to the one found in Ref. 25] $B_{d}\left(0, g_{\pi N N}\right)=4.2 \mathrm{MeV}^{3}$. Deuteron observables in the explicit $m=0$ limit are listed in Table [I]

\footnotetext{
3 These authors look for poles of the $S$-matrix so constructed as to reproduce the physical scattering length $\alpha_{0}=5.42 \mathrm{fm}$ and effective range $r_{0}=1.75 \mathrm{fm}$ at the physical value of the pion mass in the ${ }^{3} S_{1}$ eigen channel. By doing so the explicit dependence on $g_{\pi N N}$ becomes rather weak. Actually, they take $g_{\pi N N}=12.73$ and we would get instead $B_{d}\left(0, g_{\pi N N}\right)=0.98 \mathrm{MeV}$ instead. This apparent contradiction is resolved by noting that, as we will see below, for $g_{\pi N N}=13.1083$ in the $\mathrm{OPE}$ we get an scattering length $\alpha_{0}=5.335 \mathrm{fm}$ an effective range of $r_{0}=1.63$ quite close to the experimental values.
} 


\section{B. Implicit pion mass dependence}

To take into account the implicit pion mass dependence we have to take into account the dependence of $R=3 g_{A}^{2} M / 32 \pi f_{\pi}^{2}$ on the pion mass. In the chiral limit one gets a larger OPE coupling [29]. The value is uncertain and as an educated guess we take $R_{0}=1.06(2) R$. Using the same formulation as in the $m$ dependence, the change in the deuteron binding with respect to the $g_{\pi N N}$ coupling constant or equivalently the scale dimension $R$ we get (assuming as before the short distance angle $\varphi$ to be independent on $\mathrm{m}$ ),

$$
-R \frac{\partial \gamma^{2}}{\partial R}=\int_{0}^{\infty} d r\left[u_{A}(r)^{2} U_{A}+u_{R}(r)^{2} U_{R}\right]
$$

Again, the result is indefinite since $U_{A}<0$ and $U_{R}>0$ and it is not obvious, unlike naive expectations, that a stronger coupling provides stronger binding. The sign depends actually on the details of the wave functions and the particular values of the parameters. Numerically one finds $d \gamma / d R>0$, a trend that can be understood if the repulsive term in Eq. (34) is neglected or on the basis of the inequality $\left|u_{A}\right|>\left|u_{R}\right|$ which is numerically fulfilled. In any case one has the differential inequality $d \gamma / d R<$ $\gamma / R$.

Numerically, we get $\gamma_{0}=0.61(10) \mathrm{fm}^{-1}$ and hence

$$
B_{d}^{0}=15(5) \mathrm{MeV}
$$

a value compatible with the analysis of Ref. [29] $B_{d}^{0}=$ $9.6 \pm 3$ (perhaps with larger errors $[30]^{4}$ ) but in disagreement with Refs. 25, 28] where the deuteron becomes unbound for $m<90 \mathrm{MeV}$. In any case we confirm the trend of having a stronger binding of the deuteron in the chiral limit. The corresponding observables can be looked up in Table. III

\footnotetext{
${ }^{4}$ If we take $R_{0}=1.1 R$ we get $B_{d}^{0}=33 \mathrm{MeV}$.
}

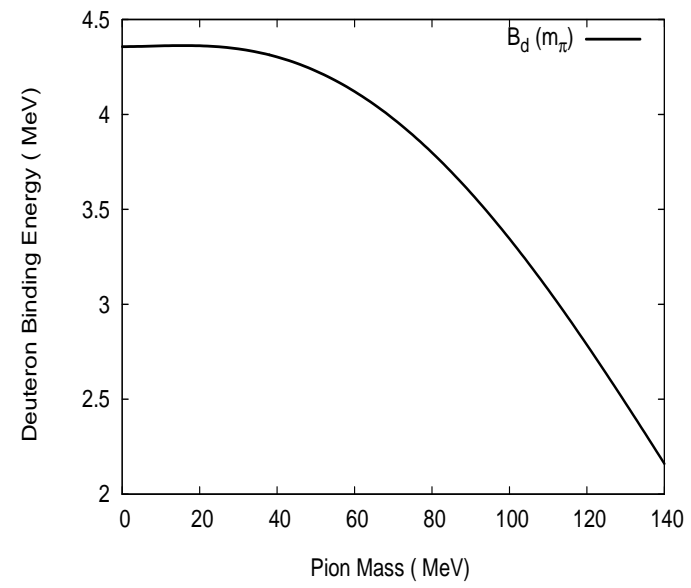

FIG. 4: Explicit pion mass dependence of the deuteron binding energy, keeping $g_{\pi N N}=13.1083$ fixed. We have $B_{d}(m)=2.22457 \mathrm{MeV}, B_{d}(0)=4.3539 \mathrm{MeV}$.

\section{SCATTERING PROPERTIES IN THE ${ }^{3} S_{1}-{ }^{3} D_{1}$ CHANNEL}

\section{A. Orthogonality constraints and Phase Shifts}

For the $\alpha$ and $\beta$ positive energy scattering states we choose the asymptotic normalization

$$
\begin{aligned}
u_{k, \alpha}(r) & \rightarrow \frac{\cos \epsilon}{\sin \delta_{1}}\left(\hat{j}_{0}(k r) \cos \delta_{1}-\hat{y}_{0}(k r) \sin \delta_{1}\right), \\
w_{k, \alpha}(r) & \rightarrow \frac{\sin \epsilon}{\sin \delta_{1}}\left(\hat{j}_{2}(k r)-\hat{y}_{2}(k r) \sin \delta_{1}\right), \\
u_{k, \beta}(r) & \rightarrow-\frac{1}{\sin \delta_{1}}\left(\hat{j}_{0}(k r) \cos \delta_{2}-y_{0}(k r) \sin \delta_{2}\right), \\
w_{k, \beta}(r) & \rightarrow \frac{\tan \epsilon}{\sin \delta_{1}}\left(\hat{j}_{2}(k r) \cos \delta_{2}-\hat{y}_{2}(k r) \sin \delta_{2}\right),
\end{aligned}
$$

where $\hat{j}_{l}(x)=x j_{l}(x)$ and $\hat{y}_{l}(x)=x y_{l}(x)$ are the reduced spherical Bessel functions and $\delta_{1}$ and $\delta_{2}$ are the eigen-phases in the ${ }^{3} S_{1}$ and ${ }^{3} D_{1}$ channels, and $\epsilon$ is the mixing angle $E_{1}$. Again, the general solution at short distances is given by the general Eq. (14), where the constants $C_{A}, C_{R}$ and $\varphi$ are now different since we have a zero energy state and depend whether we have an $\alpha$ or $\beta$ state, so we have the short distance constants, $C_{A, \alpha}(k), C_{R, \alpha}(k), \varphi_{\alpha}(k)$ and $C_{A, \beta}(k), C_{R, \beta}(k), \varphi_{\beta}(k)$ respectively. This implies certain correlations between $\delta_{1}$, $\delta_{2}$ and $\epsilon$.

For a regular self-adjoint potential the orthogonality of bound and scattering states comes out automatically. We look now for the consequences of demanding this property in the singular OPE potential. Using the standard manipulations to prove orthogonality between states of different energy we get the following relation between $\alpha$ 
TABLE II: Deuteron properties for the OPE and their dependence on the pion mass. We use the non-relativistic relation $\gamma=\sqrt{2 \mu_{n p} B}=0.231605 \mathrm{fm}^{-1}$ with $B=2.224575(9)$ for $m=138.03$ and $g_{\pi N N}=13.1083$. $m=0$ (explicit) means taking $m=0$ but using $R=1.07764 \mathrm{fm}$ (or equivalently $g_{\pi N N}=13.1083$ ). $m=0$ (implicit) means taking $m=0$ and using $R_{0}=1.06(2) \times R$.

\begin{tabular}{|l|c|c|c|c|c|c|}
\hline \hline & $\gamma\left(\mathrm{fm}^{-1}\right)$ & $\eta$ & $A_{S}\left(\mathrm{fm}^{-1 / 2}\right)$ & $r_{d}(\mathrm{fm})$ & $Q_{d}\left(\mathrm{fm}^{2}\right)$ & $P_{D}$ \\
\hline$m=138.03 \mathrm{MeV}$ & Input & 0.02633 & $0.8681(1)$ & $1.9351(5)$ & $0.2762(1)$ & $7.88(1) \%$ \\
$m=0$ (explicit) & $0.3240(1)$ & 0.09452 & $0.8444(1)$ & $1.550(1)$ & $0.3006(3)$ & $10.96(2) \%$ \\
$m=0$ (implicit) & $0.61(10)$ & $0.15(2)$ & $0.48(7)$ & $0.98(10)$ & $0.15(3)$ & $15(1) \%$ \\
\hline \hline
\end{tabular}

and $\beta$ states and the bound deuteron state (which we denote by a subscript $\gamma$ in this section),

$$
\begin{aligned}
0 & =\left(\gamma^{2}+k^{2}\right) \int_{0}^{\infty} d r\left[u_{\gamma}(r) u_{k}(r)+w_{\gamma}(r) w_{k}(r)\right] \\
& =\left.\left[u_{\gamma}^{\prime} u_{k}-u_{\gamma} u_{k}^{\prime}+w_{\gamma}^{\prime} w_{k}-w_{\gamma} w_{k}^{\prime}\right]\right|_{0} ^{\infty}
\end{aligned}
$$

Using the short distance solution, Eq. (14), we get

$$
C_{A, i}(k) C_{A}(\gamma) \sin \left[\varphi(\gamma)-\varphi_{i}(k)\right]=0 \quad, i=\alpha, \beta
$$

Which yields

$$
\varphi(\gamma)=\varphi_{\alpha}(k)=\varphi_{\beta}(k)
$$

Thus, the short distance phases $\varphi_{\alpha}(k)$ and $\varphi_{\beta}(k)$ of the ${ }^{3} S_{1}-{ }^{3} D_{1}$ channel wave functions in the OPE potential at short distances are all determined by deuteron properties. This means, in particular that the low energy parameters and scattering phase shifts are uniquely determined by the deuteron binding energy and the OPE potential parameters ${ }^{5}$.

The previous argument can also be implemented if we have a short distance cut-off at $r=a$, the orthogonality relation of Eq. (38) transforms into the condition

$$
\begin{gathered}
u_{\gamma}^{\prime}(a) u_{k, i}(a)+w_{\gamma}^{\prime}(a) w_{k, i}(a) \\
=u_{\gamma}(a) u_{k, i}^{\prime}(a)+w_{\gamma}(a) w_{k, i}^{\prime}(a) \\
i=\alpha, \beta
\end{gathered}
$$

Thus, if we impose the same condition on both solutions, Eq. (42) cannot be satisfied unless they are related at the boundary. For instance for the condition BC6 of Eqs. (15), we get the two relations

$$
u_{k, i}^{\prime}(a)=\sqrt{2} w_{k, i}^{\prime}(a), \quad i=\alpha, \beta
$$

\footnotetext{
5 This property does not hold for other triplet channels with higher partial waves, because there are no bound states in those channels. Nevertheless, it is also true that there is only one independent parameter. This means in practice that one can use one scattering length out of the three to predict the phase shifts also in other partial waves.
}

The orthogonality relation corresponding to boundary conditions of the form of Eq. (43) implies then the orthogonality constraint

$$
\frac{w_{k, i}^{\prime}(a)}{u_{k, i}(a) \sqrt{2}+w_{k, i}(a)}=\frac{w_{\gamma}^{\prime}(a)}{u_{\gamma}(a) \sqrt{2}+w_{\gamma}(a)}
$$

Which is the analog finite cut-off condition of Eq. (40). The remaining conditions in Eqs. (15) generate analogous orthogonality constraints.

The results for the ${ }^{3} S_{1}-{ }^{3} D_{1}$ channel phase shifts using these conditions are presented in Fig. [5] The description is rather satisfactory and it seems to work, as one might expect, up to the vicinity of the CM momentum which magnitude coincides with the two pion exchange left cut $k=\mathrm{i} m$.

\section{B. Low energy parameters}

In the low energy limit one has

$$
\begin{aligned}
\delta_{1} & \rightarrow-\alpha_{0} k, \\
\delta_{2} & \rightarrow-\alpha_{2} k^{5}, \\
\epsilon & \rightarrow \frac{\alpha_{02}}{\alpha_{0}} k^{2}
\end{aligned}
$$

so that the zero energy the wave functions behave asymptotically

$$
\begin{aligned}
u_{0, \alpha}(r) & \rightarrow 1-\frac{r}{\alpha_{0}}, \\
w_{0, \alpha}(r) & \rightarrow \frac{3 \alpha_{02}}{\alpha_{0} r^{2}}, \\
u_{0, \beta}(r) & \rightarrow \frac{r}{\alpha_{0}}, \\
w_{0, \beta}(r) & =\frac{3 \alpha_{2}}{\alpha_{02} r^{2}}-\frac{r^{3}}{15 \alpha_{02}} .
\end{aligned}
$$

Using these zero energy solutions one can determine the effective range. The ${ }^{3} S_{1}$ effective range parameter is given by

$$
r_{0}=2 \int_{0}^{\infty}\left[\left(1-\frac{r}{\alpha_{0}}\right)^{2}-u_{\alpha}(r)^{2}-w_{\alpha}(r)^{2}\right] d r .
$$



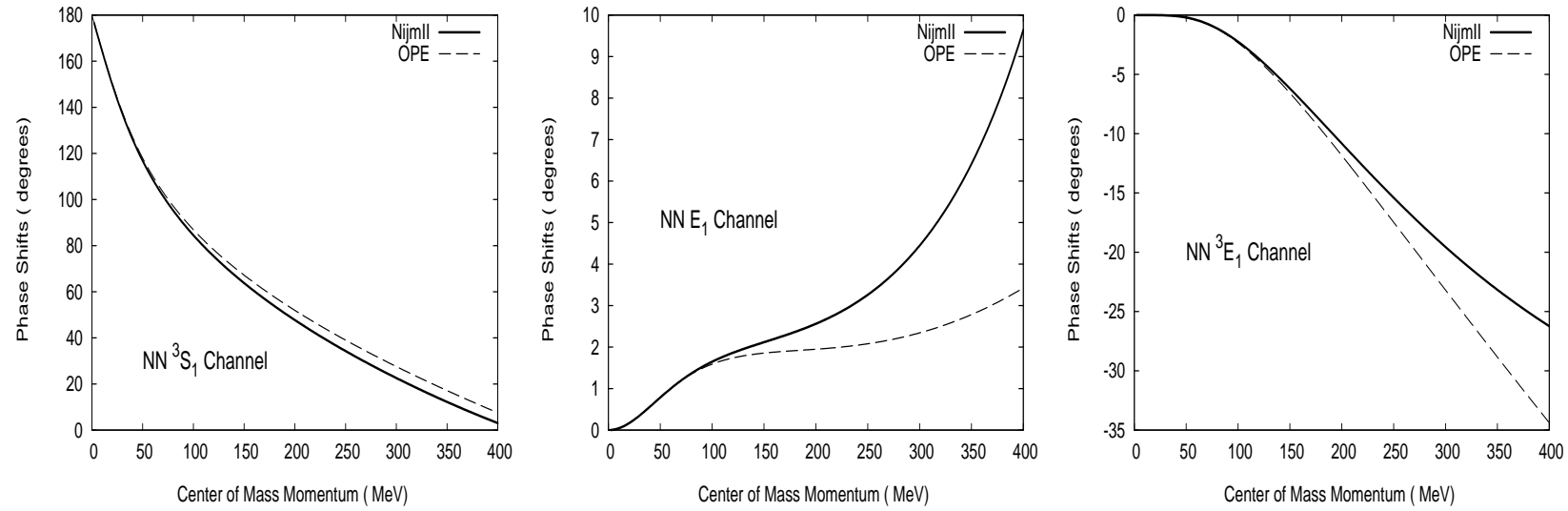

FIG. 5: Eigen Phase shifts for the OPE potential as a function of the CM np momentum in the triplet ${ }^{3} S_{1}-{ }^{3} D_{1}$ channel compared to the Nijmegen results [38]. The regular scattering wave functions are orthogonal to the regular deuteron bound state wave functions constructed from the OPE with $\gamma=0.231605 \mathrm{fm}^{-1}, m=138.03 \mathrm{MeV}$ and $g_{\pi N N}=13.1083$.

In the zero energy case, the vanishing of the diverging exponentials at the origin imposes a condition on the $\alpha$ and $\beta$ states which generate a correlation between $\alpha_{0}$, $\alpha_{02}$ and $\alpha_{2}$. Using the superposition principle of boundary conditions we may write the solutions in such a way that

$$
\begin{aligned}
u_{0, \alpha}(r) & =u_{1}(r)-\frac{1}{\alpha_{0}} u_{2}(r)+\frac{3 \alpha_{02}}{\alpha_{0}} u_{3}(r) \\
w_{0, \alpha}(r) & =w_{1}(r)-\frac{1}{\alpha_{0}} w_{2}(r)+\frac{3 \alpha_{02}}{\alpha_{0}} w_{3}(r) \\
u_{\beta}(r) & =\frac{1}{\alpha_{0}} u_{2}(r)+\frac{3 \alpha_{2}}{\alpha_{02}} u_{3}(r)-\frac{1}{15 \alpha_{02}} u_{4}(r) \\
w_{\beta}(r) & =\frac{1}{\alpha_{0}} w_{2}(r)+\frac{3 \alpha_{2}}{\alpha_{02}} w_{3}(r)-\frac{1}{15 \alpha_{02}} w_{4}(r)
\end{aligned}
$$

where the functions $u_{1,2,3,4}$ and $w_{1,2,3,4}$ are independent on $\alpha_{0}, \alpha_{02}$ and $\alpha_{2}$ and fulfill suitable boundary conditions. As a consequence we get a linear correlation between $1 / \alpha_{0}, \alpha_{02} / \alpha_{0}$ and also a linear correlation between $\alpha_{2} / \alpha_{02}$ and $1 / \alpha_{02}$. This means in turn that according to the OPE potential both $\alpha_{02}$ and $\alpha_{2}$ depends linearly with $\alpha_{0}$. Numerically we get the following correlations,

$$
\begin{aligned}
\alpha_{02} & =0.963571370240 \alpha_{0}-3.467616391389 \\
\alpha_{2} & =3.467616391389 \frac{\alpha_{02}}{\alpha_{0}}+5.080264230656
\end{aligned}
$$

These relations are cut-off independent and unique consequences of the OPE potential. On the other hand, the orthogonality between the bound state and the scattering state yields

$$
\begin{aligned}
\alpha_{0} & =1.037805911852 \alpha_{02}+3.598712446758 \\
\alpha_{02} & =0.288382561043 \alpha_{0} \alpha_{2}-1.465059639612 \alpha_{0}
\end{aligned}
$$

The provided high accuracy is indeed needed. The four equations, Eq. (48) and Eq. (49), overdetermine the values of the three scattering lengths and could be solved in triplets yielding four different solutions. Actually, there are only two independent solutions which differences are compatible within our numerical uncertainties. The scattering lengths and effective range are presented in Table $\prod$ and compared to their perturbative value ( see Appendix A and to the high quality Nijmegen potential models 33$]^{6}$. As we see, the agreement with the high quality potentials is at the few percent level. Perturbation theory does not account for most of the contribution to the effective range since the orthogonality constraints preclude a short distance contribution to $r_{0}$ and also to the deuteron matter radius $r_{m}$. This means in practice that the counterterm named $C_{2}$ in Refs. 16, 36] must vanish (See Appendix A for a detailed discussion). The dependence of the scattering lengths $\alpha_{0}, \alpha_{02}$ and $\alpha_{2}$ on the deuteron wavenumber $\gamma$ can be seen in Fig. 6] where $\gamma$ dependent generalizations of the correlations, Eq. (48) and Eq. (49) hold.

The phase shifts look very similar to previous work [25] using an energy expansion of a square well potential as a counter-term and adjusting the depth of the two lowest orders to reproduce the ${ }^{3} S_{1}$ scattering length $\alpha_{0}$ and effective range $r_{0}$ as independent parameters or our variable phase approach with non-trivial initial conditions in Ref. [35] where the full coupled channel S-matrix was tailored to reproduce the effective range expansion to any order treating all parameters as independent. Roughly speaking, both approaches could be mapped to an energy dependent boundary condition with no a priori orthogonality constraints ${ }^{7}$. The fact that the orthogonality

\footnotetext{
6 The values of $\alpha_{0}$ and $r_{0}$ have been determined in Ref. [37], whereas $\alpha_{02}$ and $\alpha_{2}$ have been determined by us in Ref. [35]. See also Ref. [39] for a extensive determination in all partial waves.

7 The main difference in this regard has to do with the multivaluation problem of the potential counter-term in Ref. [25] typical of inverse scattering problems. The approach of Ref. [35]
} 

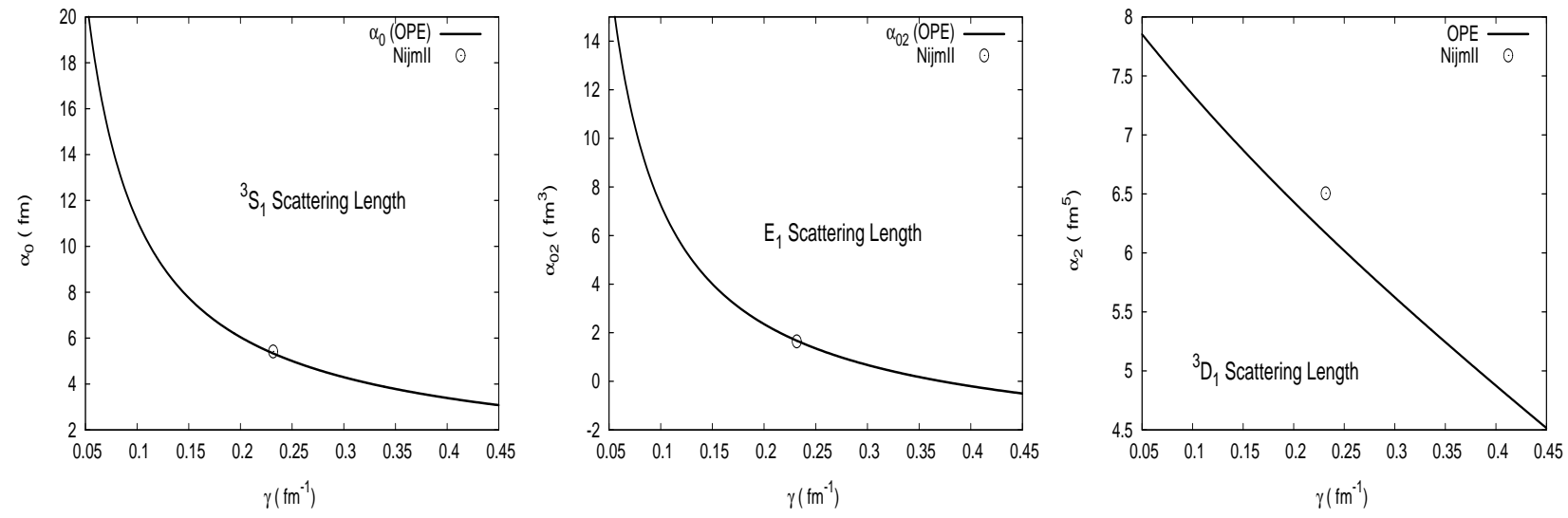

FIG. 6: The dependence of the ${ }^{3} S_{1}-{ }^{3} D_{1}$ scattering lengths $\alpha_{0}, \alpha_{02}$ and $\alpha_{2}$ on the deuteron wave number $\gamma\left(\right.$ in $\left.\mathrm{fm}^{-1}\right)$. The point represents the experimental values.

constrained boundary condition generates the bulk of the low energy threshold parameters with only one parameter naturally explains the similarity between the present phase shifts and those in previous works [25] and suggests that there is perhaps no need to make the short distance boundary condition energy dependent if the short distance cut-off is removed.

\section{SHORT DISTANCE SOLUTIONS AND DETERMINATION OF THE COEFFICIENTS}

In this section we determine the coefficients of the OPE deuteron wave functions appearing at short distances in Eq. (111). In particular, we compute the energy independent and OPE potential parameters independent short distance phase $\varphi$. Let us remind that any choice of $\varphi$ corresponds to a different choice of short distance physics; given $\varphi$ and the OPE potential all deuteron and scattering properties are uniquely determined. However, the leading asymptotic form cannot directly be used to match the numerical solution obtained by integrating in the large distance solution. On the one hand, if we use cut-off approaches to determine the regular solution, there are short distance cut-off effects when the distance gets close to the cut-off radius. On the other hand, the fact that the diverging exponential dominates over the converging one provides too weak a signal for the corresponding coefficient. To remedy the situation we improve on the short distance solution to provide a reliable approximation at larger distances $(\sim 1 \mathrm{fm})$ where the diverging exponential is less dominant, and look for plateaus in the matching radius. It turns out (see below) that one should go at eight order in this expansion for a robust determination of the short distance coefficients. Actually, one can then

does not have this problem. directly match the short distance improved wave functions to the numerical solution without no reference to cut-offs. We will try the two methods and see that they yield to compatible results for the short distance coefficients.

In the limit $r \rightarrow 0$ the solutions to the coupled equations can be written in an expansion of the form ${ }^{8}$

$$
\begin{aligned}
& u(r)=u_{0}\left(\frac{r}{R}\right)^{a_{1}} e^{a_{0} \sqrt{\frac{R}{r}}} f(r) \\
& w(r)=w_{0}\left(\frac{r}{R}\right)^{a_{2}} e^{a_{0} \sqrt{\frac{R}{r}}} g(r)
\end{aligned}
$$

with

$$
\begin{aligned}
& f(r)=\sum_{n=0}^{\infty} A_{n}\left(\frac{r}{R}\right)^{n / 2} \\
& g(r)=\sum_{n=0}^{\infty} B_{n}\left(\frac{r}{R}\right)^{n / 2}
\end{aligned}
$$

At leading order we get the equations

$$
\begin{aligned}
u_{0} a_{0}^{2}+16 \sqrt{2} w_{0} & =0 \\
16 \sqrt{2} u_{0}+\left(a_{0}^{2}-16\right) w_{0} & =0
\end{aligned}
$$

\footnotetext{
8 This expansion looks similar to a coupled channel WKB expansion but it is free of some inconveniencies. The applicability condition of the coupled channel WKB method would be that the de Broglie local wavelength matrix should be a slowly varying function of distance, implying in turn three conditions on the corresponding local wavelength eigenvalues as well as the corresponding WKB mixing angle which need not be necessarily satisfied simultaneously, generating conversion mode problems.
} 
which have the four non trivial solutions,

$$
\begin{aligned}
& (1 A), \quad a_{0}=-4 i, \quad w_{0}=\frac{u_{0}}{\sqrt{2}}, \\
& (2 A), \quad a_{0}=+4 i, \quad w_{0}=\frac{u_{0}}{\sqrt{2}}, \\
& (2 R), \quad a_{0}=-4 \sqrt{2}, \quad w_{0}=-\sqrt{2} u_{0}, \\
& (1 R), \quad a_{0}=+4 \sqrt{2}, \quad w_{0}=-\sqrt{2} u_{0} \text {. }
\end{aligned}
$$

The next to leading order equation becomes compatible only if

$$
a_{1}=a_{2}=3 / 4
$$

For any solution in Eq. (52) we may then solve for the remaining coefficients. One peculiar feature of this expansion is that if one wants to determine the solution to a given order, one has to compute the coefficients at a higher order. The reason is that strictly speaking a truncation of the expansion involves also non-diagonal elements, and one has the freedom to choose between solving $u$ or $w$ to a given accuracy. The explicit result to eight order is presented in Appendix B The general short distance solution is written as a linear combination of the four independent solutions,

$$
\begin{aligned}
u(r) & =\frac{1}{\sqrt{3}}\left(\frac{r}{R}\right)^{3 / 4}\left[-C_{1 R} f_{1 R}(r) e^{+4 \sqrt{2} \sqrt{\frac{R}{r}}}\right. \\
& -C_{2 R} f_{2 R}(r) e^{-4 \sqrt{2} \sqrt{\frac{R}{r}}}+\sqrt{2} C_{1 A} f_{1 A}(r) e^{-4 i \sqrt{\frac{R}{r}}} \\
& \left.+\sqrt{2} C_{2 A} f_{2 A}(r) e^{4 i \sqrt{\frac{R}{r}}}\right] \\
w(r) & =\frac{1}{\sqrt{3}}\left(\frac{r}{R}\right)^{3 / 4}\left[\sqrt{2} C_{1 R} g_{1 R}(r) e^{+4 \sqrt{2} \sqrt{\frac{R}{r}}}\right. \\
& +\sqrt{2} C_{2 R} g_{2 R}(r) e^{-4 \sqrt{2} \sqrt{\frac{R}{r}}}+C_{1 A} g_{1 A}(r) e^{-4 i \sqrt{\frac{R}{r}}} \\
& \left.+C_{2 A} g_{2 A}(r) e^{4 i \sqrt{\frac{R}{r}}}\right]
\end{aligned}
$$

This expansion converges rather fast for each solution up to distances of about $r \sim 0.6-0.9 \mathrm{fm}$. That is about what one needs, since that is sufficiently far above the cut-off radius $a \sim 0.1 \mathrm{fm}$. Matching $u, w, u^{\prime}$ and $w^{\prime}$ at some point in this region we get a linear relation between $C_{1 R}, C_{2 R}, C_{1 A}, C_{2 A}$ and $\eta$. Actually, we find that the signal of the converging exponential is about hundred to thousand times that of the diverging exponential in the range between 0.6 and $1 \mathrm{fm}{ }^{9}$. Matching directly the integrated in solution to the short distance solution with a vanishing coefficient of the diverging exponential $C_{1 R}=0$, we get at the scale $0.7<r<0.9 \mathrm{fm}$

$$
\begin{array}{ll}
C_{2 R}=-0.47(1), \quad \eta=0.0263333(1) \\
\bar{C}_{1 A}=0.1327(3) \quad, \bar{C}_{2 A}=0.2277(5)
\end{array}
$$

We can instead determine $\eta=0.0263332$ from the boundary condition BC6 in Eq. (15) at $r=0.2 \mathrm{fm}$ and deduce the remaining constants yielding

$$
\begin{aligned}
\left|C_{1 R}\right| & <10^{-7}, \quad C_{2 R}=-0.47(1) \\
\bar{C}_{1 A} & =0.1327(3), \quad \bar{C}_{2 A}=0.2277(5)
\end{aligned}
$$

The errors have been estimated by varying the matching point in the region $0.7 \mathrm{fm}<r<0.9 \mathrm{fm}$. Note that although the coefficient of the diverging exponential $C_{1 R}$ is six orders of magnitude larger than the one of the converging exponential, the solution through the matching condition is about eight orders of magnitude smaller (may even change sign ). So that the result provides a sizeable signal for the converging exponential. With these values we show in Fig. 7 the short distance wave functions compared to the integrated in numerical ones when the matching is undertaken at $r=0.8 \mathrm{fm}$. To improve on the short distance side we have taken $C_{1 R}=0$. The error in the region $0.7 \mathrm{fm}<r<0.9$ never exceeds a $0.01 \%$. We have checked that setting the constant $C_{2 R}=0$ introduces a larger deviation from the numerical solution as compared to the computed value in the region above $1 \mathrm{fm}$. Finally, the corresponding short distance angle reads

$$
\varphi=-\tan ^{-1} \frac{\bar{C}_{2 A}}{\bar{C}_{1 A}}=-59.7(1)^{o} .
$$

The discussion in this section explicitly shows that contrary to the findings in Ref. [11] the coefficient of the converging exponential does not vanish.

$$
\begin{aligned}
& { }^{9} \text { For instance at } r=0.8 \mathrm{fm} \text { we get } \\
& u=1.9683-59.4526 \eta \\
& =1.14054 \bar{C}_{1 A}-52.3866 C_{1 R}+1.09959 \bar{C}_{2 A}-0.00169556 C_{2 R}, \\
& w=-4.00531+159.28 \eta \\
& =-0.667631 \bar{C}_{1 A}-551.52 C_{1 R}+1.67549 \bar{C}_{2 A}+0.0177228 C_{2 R} \\
& u^{\prime}=-6.84126+287.992 \eta \\
& =-1.34949 \bar{C}_{1 a}+273.671 C_{1 R}+4.02872 \bar{C}_{2 A}-0.00925419 C_{2 R} \\
& w^{\prime}=16.2726-607.14 \eta \\
& =-0.667631 \bar{C}_{1 A}-551.52 C_{1 R}+1.67549 \bar{C}_{2 A}+0.0177228 C_{2 R}
\end{aligned}
$$

where the l.h.s. corresponds to the numerical solution and the r.h.s. to the short distance approximation, and the barred coefficients $\bar{C}_{1 A}=\left(C_{1 A}+C_{2 A}\right) / 2$ and $\bar{C}_{2 A}=\left(-C_{1 A}+C_{2 A}\right) / 2 i$ have been introduced. 


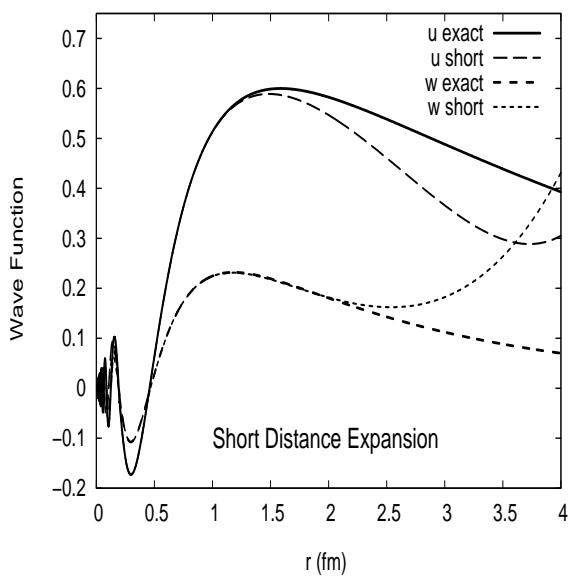

FIG. 7: The short distance expansion for the deuteron wave functions matched to the numerical solution at a distance about $r=1 \mathrm{fm}$. We take $C_{1 A}=0.1328, C_{2 A}=0.2277, C_{2 R}=$ -0.46 and $C_{1 R}=0$. The numerical solutions are normalized by taking $A_{S}=1$.

\section{CONCLUSIONS AND OUTLOOK}

In this paper we have reanalyzed the OPE potential in the triplet ${ }^{3} S_{1}-{ }^{3} D_{1}$ channel both for bound and scattering states. Rather than modeling the interaction below some finite short distance we have adopted the viewpoint of taking the potential seriously down to the origin. This must be carefully done and in a way as to get rid of any short distance ambiguities. In addition, this procedure proves crucial to be able to disentangle the OPE contribution from other contributions, like TPE and higher, electromagnetic effects and relativistic corrections to deuteron and NN scattering observables. Our analysis is carried out entirely in coordinate space where these corrections generate a potentical which is finite everywhere except at the origin. Momentum space treatments require an additional regularization of the potential.

The OPE coupled channel potential is singular at short distances and additional conditions need to be specified on the wave functions at the origin. Actually, the singular eigen-potentials at short distances are attractive and repulsive and while in the attractive case a mixed boundary condition specifies the corresponding short distance eigenfunction, in the repulsive case one must impose a standard homogeneous boundary condition. This only leaves one free parameter, which we have chosen to be the deuteron binding energy and which cannot be determined from the OPE potential. All remaining deuteron observables come out for free. For the scattering states in the ${ }^{3} S_{1}-{ }^{3} D_{1}$ channel, we have demanded orthogonality constraints between all states of different energy. This condition is actually an additional requirement for singular potentials, since the orthogonality relation carries information on the peculiar short distance behavior of the wave functions, and is not necessarily satisfied. The most obvious example where orthogonality constraints are violated corresponds to energy dependent potentials and energy dependent boundary conditions in coordinate space. A less trivial but significant example is the case of dimensional regularization in the PDS scheme as a perturbative analysis in coordinate space of both bound and scattering states reveals. The power of the orthogonality constraints for singular potentials is that all scattering properties are then predicted from the OPE potential parameters and the deuteron binding energy.

In our analysis it turns out that the short distance form of all wave functions is characterized by some short distance constants. We have clarified the role played by the exponentially suppressed regular solution by determining its non-vanishing value numerically using short distance expansions to high order, to explore the region below $0.1 \mathrm{fm}$, not accessible to standard numerical integration methods. Another relevant constant is given by a short distance phase $\varphi$ which plays the role of a fundamental dimensionless constant in the OPE problem. It does not depend on the energy nor on the OPE parameters, but it is related to the form of the OPE potential in the chiral limit. The closeness of this phase to $\pi / 3$ is mysterious and suggestive and requires further investigation.

It is remarkable that indeed the bulk of the experimental results both for the bound state as well the scattering observables are accounted for at the $2-3 \%$ level by the OPE potential taken from zero to infinity. We interpret this success as a confirmation on the validity of our choice of regular solutions and the use of orthogonality constraints. The discrepancies can legitimately be attributed to other effects such as TPE, electromagnetic and relativistic corrections. Many of the methods and results obtained in this paper can be generalized in a straightforward manner to take these effects into account and to the study of higher partial waves without any substantial modifications. In particular, the number of independent constants in a given channel depends on the short distance behavior of the long range potential. The bonus of such a program would be the complete elimination of short distance ambiguities in the study of the NN interaction with known long distance forces as determined by chiral symmetry. In our view this an indispensable prerequisite to asses the relevance of chiral symmetry in nuclear physics in a model independent way. The systematic study of these effects will be reported elsewhere [24].

\section{Acknowledgments}

We thank J. Nieves for a critical reading of the manuscript. This research was supported by DGI and FEDER funds, under contract BFM2002-03218 and by the Junta de Andalucía. 


\section{APPENDIX A: PERTURBATIVE SOLUTIONS}

\section{Bound state}

In this appendix we solve the coupled deuteron equations, Eq. (2) in standard perturbation theory for the fixed negative energy bound state. A somewhat related approach looking for the equivalence with the PDS scheme of 36 in the one-channel positive energy case can be looked up in Ref. [40]. The problem of orthogonality was not discussed. The requirement of normalizability of the deuteron state requires the $\mathrm{D}$ wave component to vanish. Thus, at lowest order we have the normalizable solutions,

$$
\begin{aligned}
u_{\gamma}^{(0)}(r) & =e^{-\gamma r} \\
w_{\gamma}^{(0)}(r) & =0
\end{aligned}
$$

At first order we have to solve the equations

$$
\begin{aligned}
-u_{\gamma}^{(1) \prime \prime}(r)+\gamma^{2} u_{\gamma}^{(1)}(r) & =-U_{s}(r) e^{-\gamma r} \\
-w_{\gamma}^{(1) \prime \prime}(r)+\left[\frac{6}{r^{2}}+\gamma^{2}\right] w_{\gamma}^{(1)}(r) & =-U_{s d}(r) e^{-\gamma r},
\end{aligned}
$$

Using the regular and irregular solutions at the origin

$$
\begin{aligned}
u_{\text {reg }}(r) & =2 \frac{\sinh (\gamma r)}{\gamma r} \\
w_{\text {reg }}(r) & =2\left(1+\frac{3}{(\gamma r)^{2}}\right) \sinh (\gamma r)-\frac{6}{\gamma r} \cosh (\gamma r) \\
u_{\text {irreg }}(r) & =e^{-\gamma r} . \\
w_{\text {irreg }}(r) & =e^{-\gamma r}\left(1+\frac{3}{\gamma r}+\frac{3}{(\gamma r)^{2}}\right)
\end{aligned}
$$

we get

$$
\begin{aligned}
& u_{\gamma}^{(1)}(r)=\int_{0}^{\infty} G_{s}\left(r, r^{\prime}\right) U_{s}\left(r^{\prime}\right) e^{-\gamma r^{\prime}} d r^{\prime} \\
& w_{\gamma}^{(1)}(r)=\int_{0}^{\infty} G_{d}\left(r, r^{\prime}\right) U_{s d}\left(r^{\prime}\right) e^{-\gamma r^{\prime}} d r^{\prime}
\end{aligned}
$$

where $G_{s}$ and $G_{d}$ are the corresponding Green functions. Explicit calculation yields

$$
\begin{aligned}
& u_{\gamma}^{(1)}(r)=e^{-\gamma r} \frac{m^{2} R \Gamma(0, m r+2 r \gamma)-\Gamma(0, m r)}{3 \gamma}-\frac{2 m^{2} R \operatorname{Ei}(-m r-2 r \gamma) \sinh (r \gamma)}{3 \gamma} \\
& w_{\gamma}^{(1)}(r)=e^{-r \gamma}\left(1+\frac{3}{r^{2} \gamma^{2}}+\frac{3}{r \gamma}\right) \times \\
& {\left[\frac{m^{2} R\left(3 m^{2}-4 \gamma^{2}\right) \Gamma(0, m r+2 r \gamma)-\Gamma(0, m r)}{6 \sqrt{2} \gamma^{3}}\right.} \\
& +\frac{R\left(-6 m^{3} \gamma+6 m^{2} \gamma^{2}+4 \gamma^{4}+\left(3 m^{4}-4 m^{2} \gamma^{2}\right) \log \left(1+\frac{2 \gamma}{m}\right)\right)}{6 \sqrt{2} \gamma^{3}} \\
& +\frac{e^{-m r-2 r \gamma} R\left(6+6 m r+m^{2} r^{2}-m^{3} r^{3}+4 r \gamma+4 m r^{2} \gamma+2 m^{2} r^{3} \gamma\right)}{2 \sqrt{2} r^{4} \gamma^{3}} \\
& \left.-\frac{R e^{-m r}\left(6+6 m r+m^{2} r^{2}-m^{3} r^{3}-8 r \gamma-8 m r^{2} \gamma+4 r^{2} \gamma^{2}+4 m r^{3} \gamma^{2}\right)}{2 \sqrt{2} r^{4} \gamma^{3}}\right] \\
& +\left(2\left(1+\frac{3}{\gamma^{2} r^{2}}\right) \sinh (\gamma r)-\frac{6}{\gamma r} \cosh (\gamma r)\right) \times \\
& {\left[\frac{e^{-m r-2 r \gamma} R\left(6+6 m r+m^{2} r^{2}-m^{3} r^{3}+4 r \gamma+4 m r^{2} \gamma+2 m^{2} r^{3} \gamma\right)}{2 \sqrt{2} r^{4} \gamma^{3}}\right.} \\
& \left.-\frac{m^{2} R\left(3 m^{2}-4 \gamma^{2}\right) \operatorname{Ei}(-m r-2 r \gamma)}{6 \sqrt{2} \gamma^{3}}\right]
\end{aligned}
$$

where $\Gamma(0, z)$ and $\operatorname{Ei}(z)$ are the standard incomplete Gamma function and the Exponential integral function respectively

$$
\begin{aligned}
\Gamma(0, z) & =\int_{z}^{\infty} d t \frac{e^{-t}}{t} \\
\operatorname{Ei}(z) & =-P \int_{-z}^{\infty} d t \frac{e^{-t}}{t}
\end{aligned}
$$


At asymptotically large distances we have

$$
\begin{aligned}
u_{\gamma}^{(1)}(r) & \rightarrow c_{\text {pert }} e^{-\gamma r} \\
w_{\gamma}^{(1)}(r) & \rightarrow \eta_{\text {pert }} e^{-\gamma r}\left(1+\frac{3}{\gamma r}+\frac{3}{(\gamma r)^{2}}\right)
\end{aligned}
$$

where

$$
\begin{aligned}
& c_{\text {pert }}=\int_{0}^{\infty} U_{s}(r) u_{\mathrm{reg}}(r) e^{-\gamma r} d r \\
& \eta_{\text {pert }}=\int_{0}^{\infty} U_{s d}(r) w_{\mathrm{reg}}(r) e^{-\gamma r} d r
\end{aligned}
$$

Explicit calculation yields

$$
\begin{aligned}
c_{\text {pert }} & =\frac{R m^{2}}{3 \gamma} \log \left(1+\frac{2 \gamma}{m}\right) \\
\eta_{\text {Pert }} & =\frac{R}{6 \sqrt{2} m \gamma^{3}}\left[4 \gamma^{4}+6 m^{2} \gamma^{2}-6 m^{3} \gamma\right. \\
& \left.+\left(3 m^{4}-4 m^{2} \gamma^{2}\right) \log \left(1+\frac{2 \gamma}{m}\right)\right] \\
& =\frac{32 \sqrt{2} R}{45 m} \gamma^{2}-\frac{2 \sqrt{2} R \gamma^{3}}{3 m^{2}}+\ldots
\end{aligned}
$$

The numerical value we get is $\eta_{\text {pert }}=0.0510$ almost twice the exact OPE result. Taking this perturbative value for $\eta$ we show in Fig. 8 the perturbative deuteron wave functions as compared to the exact ones.

Unfortunately, if one wants to improve on this first order calculation going to second order perturbation theory there is a problem since the behavior of the perturbative wave functions at short distances is given by

$$
\begin{aligned}
& u_{\gamma}^{(1)}(r)=-\frac{R m^{2}}{3 \gamma} \log \left(1+\frac{2 \gamma}{m}\right)+\ldots \\
& w_{\gamma}^{(1)}(r)=\frac{\sqrt{2} R}{r}-\frac{2}{3} \sqrt{2} R \gamma+\ldots
\end{aligned}
$$

making the wave function non normalizable, unlike the exact regular wave function. This divergence at short distances actually precludes going to higher orders in perturbation theory.

The normalization at first order is given by

$$
\frac{1}{A_{S}^{2}}=\int_{0}^{\infty}\left(e^{-2 \gamma r}+2 u_{\gamma}^{(1)}(r) e^{-\gamma r}\right)
$$

and hence

$$
\begin{aligned}
\frac{A_{S}}{\sqrt{2 \gamma}} & =1-\frac{2 R m^{2}}{3(m+2 \gamma)}+\frac{R m^{2}}{3 \gamma} \log \left(1+\frac{2 \gamma}{m}\right) \\
& =1-\frac{2 R \gamma}{3}-\frac{16 R \gamma^{2}}{9 m}+\ldots
\end{aligned}
$$

The deuteron matter radius is given by

$$
r_{m, \text { pert }}^{2}=\frac{1}{4} A_{S}^{2} \int_{0}^{\infty} r^{2}\left(e^{-2 \gamma r}+2 u_{\gamma}^{(1)}(r) e^{-\gamma r}\right)
$$

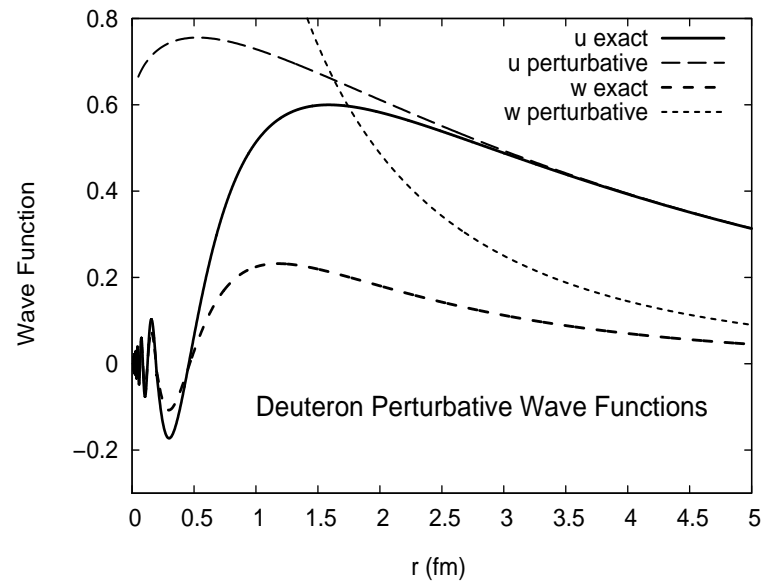

FIG. 8: Perturbative deuteron wave functions compared to the exact ones as a function of the distance (in $\mathrm{fm}$ ). The exact ones are normalized by taking $A_{S}=1$.

and hence to first order one has

$$
r_{m, \text { pert }}^{2}=\frac{1}{8 \gamma^{2}}+\frac{m^{2} R(3 m+10 \gamma)}{18 \gamma(m+2 \gamma)^{3}}+\ldots
$$

yielding in the weak binding regime

$$
\sqrt{8} \gamma r_{m, \text { pert }}=1+\frac{2 R \gamma}{3}-\frac{16 R \gamma^{2}}{9 m}+\ldots
$$

Finally, the quadrupole moment at first order is given by

$$
Q_{\text {pert }}=\frac{\sqrt{2}}{10} \int_{0}^{\infty} r^{2} w_{\gamma}^{(1)}(r) e^{-\gamma r} d r
$$

The integral can be evaluated to give

$$
\begin{aligned}
Q_{\text {pert }} & =\frac{8 R\left(4 m^{2}+9 \gamma m+6 \gamma^{2}\right)}{45(m+2 \gamma)^{3}} \\
& =\frac{32 R}{45 m}-\frac{8 R \gamma}{3 m^{2}}+\frac{128 R \gamma^{2}}{15 m^{3}}+\ldots
\end{aligned}
$$

yielding $Q_{\text {pert }}=0.4555 \mathrm{fm}^{2}$.

Our perturbative expressions for $A_{S}, r_{m}$ and $Q$ coincide with those of Kaplan Savage and Wise [16] provided one takes in their expression for $r_{m}$ the renormalization scale in the PDS scheme to be $\mu=\gamma$, instead of taking $\mu=m$ as they do or else taking the $C_{2}$ counter-term identically equal to zero. Actually, the $C_{2}$ counter-term can be mapped into a short distance contribution to the effective range parameter $r_{0}$ in the ${ }^{3} S_{1}$ channel. The value of $\eta$ was not given in that reference but can be deduced from the off-diagonal scattering amplitude in the ${ }^{3} S_{1}-{ }^{3} D_{1}$ channel given in their previous work 36 by evaluating the residue at the deuteron pole. The result also agrees with the calculation presented here. 


\section{Low energy parameters}

To check the identification $C_{2}=0$ further let us compute the S-wave effective range $r_{0}$. For our purposes it is sufficient to analyze the zero energy scattering state. The lowest order solution is given by an $\alpha$ state

$$
\begin{aligned}
& u_{0, \alpha}^{(0)}(r)=\left(1-\frac{r}{\alpha_{0}}\right) \\
& w_{0, \alpha}^{(0)}(r)=0
\end{aligned}
$$

At zeroth order in the OPE coupling the orthogonality constraint yields

$$
\begin{aligned}
0 & =\int_{0}^{\infty} u_{0, \alpha}^{(0)}(r) u_{\gamma}^{(0)}(r) d r \\
& =\frac{1}{\gamma^{2}}\left[-\frac{1}{\alpha_{0}}+\gamma\right]
\end{aligned}
$$

which yields the scattering length to lowest order,

$$
\alpha_{0}^{(0)}=\frac{1}{\gamma}
$$

At first order we use the regular solution $u_{\mathrm{reg}}(r)=r$ and the irregular solution $u_{\text {irreg }}(r)=\left(1-r / \alpha_{0}\right)$ and get similarly to the bound state case the first order correction to the $\alpha$ state,

$$
\begin{aligned}
u_{0, \alpha}^{(1)}(r) & =\frac{2 R}{3 \alpha_{0}} e^{-m r}\left(1-\alpha_{0} m\right)-\frac{2 m^{2} r R}{3} \operatorname{Ei}(-m r) \\
w_{0, \alpha}^{(1)}(r) & =\frac{e^{-m r} R}{15 \sqrt{2} \alpha_{0} m^{2} r^{2}} \\
& \times\left(120-64 \alpha_{0} m+120 m r-34 \alpha_{0} m^{2} r\right. \\
& \left.+40 m^{2} r^{2}-2 \alpha_{0} m^{3} r^{2}+\alpha_{0} m^{4} r^{3}-\alpha_{0} m^{5} r^{4}\right) \\
& +\frac{R\left(-120+64 \alpha_{0} m+\alpha_{0} m^{6} r^{5} \Gamma(0, m r)\right)}{15 \sqrt{2} \alpha_{0} m^{2} r^{2}}
\end{aligned}
$$

Note that asymptotically the first order correction to the S-wave vanishes exponentially and hence cannot contribute to the scattering length. On the other hand, the orthogonality relation to first order reads

$$
0=\int_{0}^{\infty} d r\left[u_{\gamma}^{(0)} u_{0, \alpha}^{(0)}+u_{\gamma}^{(1)} u_{0, \alpha}^{(0)}+u_{\gamma}^{(1)} u_{0, \alpha}^{(0)}\right]
$$

and after computing the integrals one gets

$$
\begin{aligned}
0=-\frac{1}{\alpha_{0}}+\gamma & +\frac{R}{3 \alpha_{0} \gamma}\left[m^{2}\left(1+\alpha_{0} \gamma\right) \log \left(1+\frac{2 \gamma}{m}\right)\right. \\
& \left.-2 \gamma\left(m-\gamma+\alpha_{0} m \gamma\right)\right]
\end{aligned}
$$

Solving perturbatively for $\alpha_{0}$ we get at first order

$$
\alpha_{0, \text { pert }}=\frac{1}{\gamma}-\frac{2 m^{2} R}{3 \gamma^{2}}\left[\frac{\gamma(\gamma-2 m)}{m^{2}}+\log \left(1+\frac{2 \gamma}{m}\right)\right]+\ldots
$$

Numerically one gets $\alpha_{0, \text { pert }}=(4.3177+0.2912+\ldots) \mathrm{fm}$ to be compared with the full OPE result $\alpha_{0}=5.34$ and the experimental value $\alpha_{0}=5.42 \mathrm{fm}$. The $E_{1}$ scattering length $\alpha_{02}$ can be read off from the D-wave, using the asymptotic condition in Eq. 46]

$$
\begin{aligned}
\alpha_{02} & =\frac{4 \sqrt{2} R\left(15-8 \alpha_{0} m\right)}{45 m^{2}} \\
& =\frac{4 \sqrt{2} R(15 \gamma-8 m)}{45 \gamma m^{2}}
\end{aligned}
$$

in the second line we have substituted the perturbative relation $\alpha_{0}=1 / \gamma+\mathcal{O}(R)$. Note the linear correlation $\alpha_{02}=1.5499 \alpha_{0}-4.1530$ to be compared with the exact $\mathrm{OPE}$ relation in Eq. (48). The numerical value one gets for the first and second lines taking the experimental values of $\alpha_{0}=5.42$ and $\gamma$ are $\alpha_{02}=4.24 \mathrm{fm}^{3}$ and $\alpha_{02}=2.53 \mathrm{fm}^{3}$ respectively to be compared with the experimental $\alpha_{02}=1.64 \mathrm{fm}^{3}$. In the weak binding limit one obtains

$$
\gamma \alpha_{0, \text { pert }}=1+\frac{2 R \gamma}{3}-\frac{16 R \gamma^{2}}{9 m}+\ldots
$$

In this limit we have the perturbative linear correlation between the scattering length and the deuteron matter radius

$$
r_{m}=\frac{\alpha_{0}}{2 \sqrt{2}}+\mathcal{O}\left(\gamma^{3}, R^{2}\right)
$$

which yields the value $r_{m}=1.92$ for the experimental scattering length $\alpha_{0}=5.42 \mathrm{fm}$. The linear correlation was established empirically with realistic potentials in Ref. [1, 42].

To first order the effective range in the ${ }^{3} S_{1}$ eigenchannel is given by

$$
r_{0}=-4 \int_{0}^{\infty} d r u_{0, \alpha}^{(0)}(r) u_{0, \alpha}^{(1)}(r)
$$

yielding

$$
\begin{aligned}
r_{0, \text { pert }} & =\frac{4 R\left(3 m^{2}-8 \gamma m+6 \gamma^{2}\right)}{9 m^{2}} \\
& =1.4369-5.4789 \gamma+5.8758 \gamma^{2} \\
& =0.4831 \mathrm{fm}
\end{aligned}
$$

a result much smaller than the full OPE result $(1.64 \mathrm{fm})$ and the experimental number $(1.75 \mathrm{fm})$. Again, our result corresponds to a theory where the short distance contribution to the effective range vanishes, i.e. $C_{2}=0$. A non vanishing value of $C_{2}$ was needed to fit the experimental values of both the matter radius and the effective range. Our calculation shows that the scheme developed in Refs. [16] and Ref. [36] does not fulfill perturbatively the orthogonality constraints.

\section{APPENDIX B: SHORT DISTANCE EXPANSION}

For the $f(r)$ function we get (we use $x=r / R$ ), 


$$
\begin{aligned}
f_{1 A} & =1-\frac{35 i}{32} \sqrt{x}-\frac{1811 x}{6144}+\frac{2441 i}{65536} x^{\frac{3}{2}}-\frac{34805 x^{2}}{8388608} \\
& +x^{3}\left(\frac{9873675}{17179869184}+\frac{m^{2} R^{2}}{36}-\frac{m^{3} R^{3}}{32}-\frac{3 R^{2} \gamma^{2}}{64}\right) \\
& +x^{\frac{7}{2}}\left(\frac{193405905 i}{549755813888}+\frac{353 i}{24192} m^{2} R^{2}-\frac{709 i}{92160} m^{3} R^{3}+\frac{i}{28} m^{4} R^{4}-\frac{709 i}{61440} R^{2} \gamma^{2}\right) \\
& +x^{\frac{5}{2}}\left(\frac{-333725 i}{268435456}-\frac{i}{15} m^{3} R^{3}-\frac{i}{10} R^{2} \gamma^{2}\right) \\
f_{2 A} & =f_{1 A}^{*} \\
f_{2 R} & =1+\frac{67 \sqrt{x}}{32 \sqrt{2}}+\frac{7763 x}{12288}+\left(\frac{8873}{131072 \sqrt{2}}-\frac{m^{2} R^{2}}{3 \sqrt{2}}\right) x^{\frac{3}{2}}+\left(-\frac{105845}{33554432}-\frac{55 m^{2} R^{2}}{192}\right) x^{2} \\
& +\left(\frac{881405}{1073741824 \sqrt{2}}-\frac{10807 m^{2} R^{2}}{184320 \sqrt{2}}+\frac{m^{3} R^{3}}{15 \sqrt{2}}+\frac{R^{2} \gamma^{2}}{10 \sqrt{2}}\right) x^{\frac{5}{2}} \\
& +\left(-\frac{23360715}{137438953472}-\frac{332899 m^{2} R^{2}}{11796480}+\frac{47 m^{3} R^{3}}{960}+\frac{m^{4} R^{4}}{36}+\frac{47 R^{2} \gamma^{2}}{640}\right) x^{3} \\
& +\left(\frac{419268465}{4398046511104 \sqrt{2}}+\frac{30559591 m^{2} R^{2}}{31708938240 \sqrt{2}}+\frac{2141 m^{3} R^{3}}{1290240 \sqrt{2}}+\frac{229 m^{4} R^{4}}{8064 \sqrt{2}}+\frac{2141 R^{2} \gamma^{2}}{860160 \sqrt{2}}\right) x^{\frac{7}{2}} \\
f_{1 R} & =f_{2 R}\left(x \rightarrow e^{2 \pi i} x\right)
\end{aligned}
$$

and for the $g(r)$ function one has

$$
\begin{aligned}
g_{1 A} & =1-\frac{35 i}{32} \sqrt{x}-\frac{4883 x}{6144}+\frac{82075 i}{196608} x^{\frac{3}{2}}+\frac{1245195 x^{2}}{8388608} \\
& +\left(\frac{-5136285 i}{268435456}-\frac{i}{15} m^{3} R^{3}-\frac{i}{10} R^{2} \gamma^{2}\right) x^{\frac{5}{2}} \\
& +\left(\frac{42237195}{17179869184}-\frac{m^{2} R^{2}}{18}-\frac{m^{3} R^{3}}{32}-\frac{3 R^{2} \gamma^{2}}{64}\right) x^{3} \\
& +\left(\frac{494999505 i}{549755813888}-\frac{65 i}{12096} m^{2} R^{2}+\frac{2363 i}{92160} m^{3} R^{3}+\frac{i}{28} m^{4} R^{4}+\frac{2363 i}{61440} R^{2} \gamma^{2}\right) x^{\frac{7}{2}}+\mathcal{O}\left(x^{4}\right) \\
g_{2 A} & =g_{1 A}^{*} \\
g_{2 R} & =1+\frac{67 \sqrt{x}}{32 \sqrt{2}}+\frac{13907 x}{12288}+\left(\frac{307195}{393216 \sqrt{2}}-\frac{m^{2} R^{2}}{3 \sqrt{2}}\right) x^{\frac{3}{2}}+\left(\frac{5075595}{33554432}-\frac{55 m^{2} R^{2}}{192}\right) x^{2} \\
& +\left(\frac{19661565}{1073741824 \sqrt{2}}-\frac{41527 m^{2} R^{2}}{184320 \sqrt{2}}+\frac{m^{3} R^{3}}{15 \sqrt{2}}+\frac{R^{2} \gamma^{2}}{10 \sqrt{2}}\right) x^{\frac{5}{2}} \\
& +\left(-\frac{143137995}{137438953472}-\frac{128033 m^{2} R^{2}}{3932160}+\frac{47 m^{3} R^{3}}{960}+\frac{m^{4} R^{4}}{36}+\frac{47 R^{2} \gamma^{2}}{640}\right) x^{3} \\
& +\left(\frac{1476620145}{4398046511104 \sqrt{2}}-\frac{45736601 m^{2} R^{2}}{31708938240 \sqrt{2}}+\frac{45149 m^{3} R^{3}}{1290240 \sqrt{2}}+\frac{229 m^{4} R^{4}}{8064 \sqrt{2}}+\frac{45149 R^{2} \gamma^{2}}{860160 \sqrt{2}}\right) x^{\frac{7}{2}}+\mathcal{O}\left(x^{4}\right) \\
g_{1 R} & =g_{2 R}\left(x \rightarrow e^{2 \pi i} x\right)
\end{aligned}
$$

\section{APPENDIX C: LOCAL DIAGONALIZATION AND PERTURBATIVE MIXING}

One of the puzzles one encounters in the description of the deuteron with the OPE potential is that while the dimensionless $\mathrm{D} / \mathrm{S}$ ratio parameter is rather small at long distances $w / u \rightarrow \eta=0.0256$, it actually comes from a strong mixing at short distances where $w / u \rightarrow 1 / \sqrt{2} \sim$ 0.707. Actually, the analog question for scattering states is that there seems to be a natural hierarchy for the phase shifts in the ${ }^{3} S_{1}-{ }^{3} D_{1}$ channel, namely $\delta_{3 S 1} \gg \delta_{3 D 1} \gg \epsilon_{1}$ even though the threshold behavior of the $\mathrm{D}$-wave is more suppressed than that of the mixing angle. The question is whether one can think of an expansion in terms of the 
$\eta$ parameter. There are two obvious situations where the mixing does not occur. One is the absence of tensor force. In the OPE potential that would also eliminate the $D-$ wave. Another situation is dropping the mixing terms in the OPE potential, which is questionable since they are actually larger than the diagonal terms. It is possible, however, to write the equations in a form that the mixing is manifestly small at all distances. To this end we make a local rotation of the deuteron wave functions

$$
\left(\begin{array}{c}
u(r) \\
w(r)
\end{array}\right)=\left(\begin{array}{cc}
\cos \theta(r) & \sin \theta(r) \\
-\sin \theta(r) & \cos \theta(r)
\end{array}\right)\left(\begin{array}{l}
u_{A}(r) \\
u_{R}(r)
\end{array}\right)
$$

in such a way as to diagonalize the potential we have

$$
\begin{array}{ll}
\left(\begin{array}{cc}
U_{s} & U_{s d} \\
U_{s d} & U_{d}+\frac{6}{r^{2}}
\end{array}\right) & = \\
\left(\begin{array}{cc}
\cos \theta & \sin \theta \\
-\sin \theta & \cos \theta
\end{array}\right) & \left(\begin{array}{cc}
U_{A} & 0 \\
0 & U_{R}
\end{array}\right)\left(\begin{array}{cc}
\cos \theta & \sin \theta \\
-\sin \theta & \cos \theta
\end{array}\right)
\end{array}
$$

The deuteron equations for the OPE potentials read after the local rotation

$$
\begin{aligned}
-u_{A}^{\prime \prime}(r)+\left[U_{A}(r)+\theta^{\prime}(r)^{2}\right] u_{A}(r)+\gamma^{2} u_{A}(r) & =\left[2 \theta^{\prime}(r) u_{R}^{\prime}(r)+\theta^{\prime \prime}(r) u_{R}(r)\right], \\
-u_{R}^{\prime \prime}(r)+\left[U_{R}(r)+\theta^{\prime}(r)^{2}\right] u_{R}(r)+\gamma^{2} u_{R}(r) & =-\left[2 \theta^{\prime}(r) u_{A}^{\prime}(r)+\theta^{\prime \prime}(r) u_{A}(r)\right],
\end{aligned}
$$

In the coupled channel space these equations can be visualized as a particle with spin in the presence of a gauge potential $\theta^{\prime}(r)$. At long distances we have the expansions

$$
\begin{aligned}
\theta & =\frac{2 \sqrt{2} R}{9 r} e^{-m r}\left(m^{2} r^{2}+3 m r+3\right)+\ldots \\
U_{A} & =-\frac{2 m^{2} R}{3 r} e^{-m r}+\ldots \\
U_{R} & =\frac{6}{r^{2}}+\frac{2 R}{3 r^{3}}\left(m^{2} r^{2}+6 m r+6\right) e^{-m r}+\ldots
\end{aligned}
$$

whereas at short distances we have the behavior

$$
\begin{aligned}
\theta= & \cos ^{-1} \sqrt{\frac{2}{3}}-\frac{r}{3 \sqrt{2} R}+\frac{r^{2}}{18 \sqrt{2} R^{2}} \\
& -\frac{\left(18 m^{2} R^{2}-5\right) r^{3}}{324 \sqrt{2} R^{3}}+\ldots \\
U_{A}= & -\frac{4 R}{r^{3}}+\frac{2}{r^{2}}-\frac{2}{3 r R}+\ldots \\
U_{R}= & \frac{8 R}{r^{3}}+\frac{4}{r^{2}}+\frac{2-6 m^{2} R^{2}}{3 R r}+\ldots
\end{aligned}
$$

Note that in the locally rotated basis the mixing is related to the derivative of the mixing angle, $\theta^{\prime}$ which is small at all distances (See Fig. 9). Actually, at asymptotically large distances we have

$$
u_{A}(r) \rightarrow u(r) \quad u_{R}(r) \rightarrow w(r)
$$

If we neglect the mixing term in Eq. (C3) the equations decouple and, actually, there is no non-trivial solution for the repulsive eigen-channel, since the energy is fixed arbitrarily. Hence in the absence of mixing we have $u_{R}=$ 0 . At this level of approximation we then get

$$
\begin{aligned}
u(r) & =\cos \theta(r) u_{A}(r) \\
w(r) & =\sin \theta(r) u_{A}(r)
\end{aligned}
$$

In Fig. 10 we show the solutions of the decoupled equations compared to the exact ones. As we see, the difference in the wave functions and hence the $D / S$ mixing is indeed small. Note that this is not the same as to neglect the tensor force. The results for the deuteron observables are presented in Table III As we see, the quality of the zeroth $\eta$ approximation is rather good.

Actually, we can check a posteriori that the mixing is indeed small for the zeroth order solutions. The inhomogeneous term at short distances behaves as

$$
2 \theta^{\prime}(r) u_{A}^{\prime}(r) \rightarrow-\frac{2}{3 \sqrt{2} R^{2}}\left(\frac{r}{R}\right)^{-3 / 4} C_{A} \sin (4 \sqrt{R / r}+\alpha)
$$

which compared to the remaining terms in Eq. (C3) can indeed be considered small. Under these circumstances, the mixing can then be included perturbatively, yielding

$u_{R}(r)=\int_{0}^{\infty} d r^{\prime} G_{R}\left(r, r^{\prime}\right)\left[2 \theta^{\prime}\left(r^{\prime}\right) u_{A}^{\prime}\left(r^{\prime}\right)+\theta^{\prime \prime}\left(r^{\prime}\right) u_{A}\left(r^{\prime}\right)\right]$

with $G_{R}\left(r, r^{\prime}\right)$ the Green function of the homogeneous equation in the repulsive eigen-channel,

$$
\begin{aligned}
G_{R}\left(r, r^{\prime}\right) & =w_{\text {reg }}(r) w_{\text {irreg }}\left(r^{\prime}\right) \theta\left(r^{\prime}-r\right) \\
& +w_{\text {reg }}\left(r^{\prime}\right) w_{\text {irreg }}(r) \theta\left(r-r^{\prime}\right)
\end{aligned}
$$

with Wronskian equal to unity and $w_{\text {reg }}(r)$ and $w_{\text {irreg }}(r)$ the regular solution and irregular solutions at the origin 

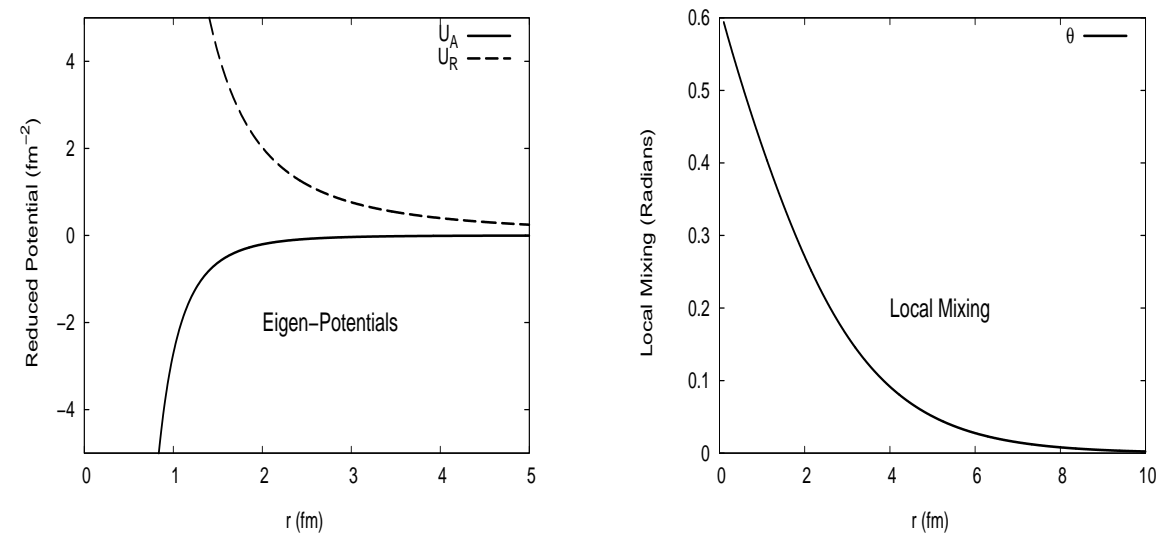

FIG. 9: (Left) Reduced eigen potentials (in $\left.\mathrm{fm}^{-2}\right) U_{A}(r)$ and $U_{R}(r)$ as a function of the distance $r$ (in fm). (Right) Local mixing angle $\theta(r)$ as a function of the distance $r$ (in $\mathrm{fm}$ ).
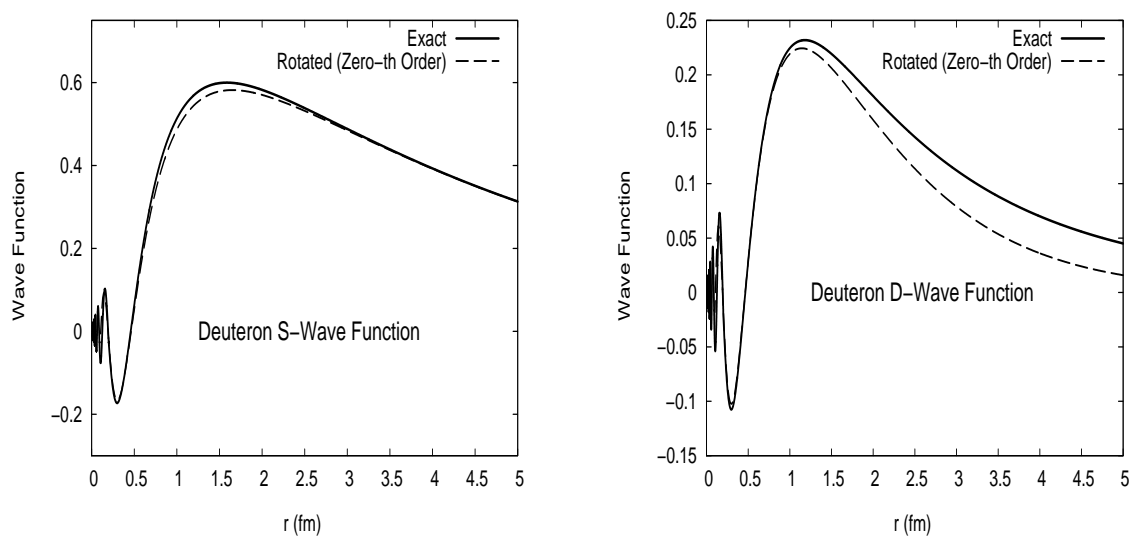

FIG. 10: The rotated eigenfunctions at zeroth order compared to the exact ones. They correspond to take $\eta=0$.

respectively. Asymptotically one has,

$$
\begin{array}{ccr}
w_{\text {reg }}(r) \rightarrow C\left(\frac{r}{R}\right)^{3 / 4} e^{-4 \sqrt{2} \sqrt{R / r}} & (r \rightarrow 0) \\
w_{\text {reg }}(r) \rightarrow e^{+\gamma r}\left(1-\frac{3}{\gamma r}+\frac{3}{(\gamma r)^{2}}\right) & (r \rightarrow \infty) \\
w_{\text {irreg }}(r) \rightarrow C\left(\frac{r}{R}\right)^{3 / 4} e^{+4 \sqrt{2} \sqrt{R / r}} & (r \rightarrow 0) \\
w_{\text {irreg }}(r) \rightarrow e^{-\gamma r}\left(1+\frac{3}{\gamma r}+\frac{3}{(\gamma r)^{2}}\right) & (r \rightarrow \infty)
\end{array}
$$

To get in practice the coefficient $C$ we start with $C=1$ at short distances and build the ratio to the asymptotic form at a sufficiently large distance. With these conditions the solution $u_{R}(r)$ at large distances behaves as

$$
u_{R}(r) \rightarrow \eta e^{-\gamma r}\left(1+\frac{3}{\gamma r}+\frac{3}{(\gamma r)^{2}}\right) \quad(r \rightarrow \infty)
$$

with

$$
\eta=\int_{0}^{\infty} d r w_{\mathrm{reg}}(r)\left[2 \theta^{\prime}(r) u_{A}^{\prime}(r)+\theta^{\prime \prime}(r) u_{A}(r)\right]
$$

At short distances we get, from Eq. (C14) and using the asymptotic forms of Eq. (C16) and Eq. (C9), the result

$$
u_{R}(r) \rightarrow C C_{A}\left(\frac{r}{R}\right)^{7 / 4} \cos (4 \sqrt{R / r}+\alpha)
$$

in agreement with the leading short distance behavior of the full solution for the combination $u(r)-\sqrt{2} w(r)$ (see Sect. VI). The perturbative value for the asymptotic $D / S$ ratio we get is

$$
\eta_{\text {pert }}=0.0261
$$

quite close to the $\mathrm{OPE}$ exact one, $\eta_{\mathrm{OPE}}=0.0263$.

\section{APPENDIX D: LONG DISTANCE SOLUTIONS}

As a complement to the perturbative treatment of Appendix $\AA$ we analyze the bound solutions at long dis- 
TABLE III: Deuteron properties for the OPE potential. We use the non-relativistic relation $\gamma=\sqrt{2 \mu_{n p} B}$ with $B=2.224575(9)$. We compare the $\eta$-expansion at leading order (LO), with standard perturbation theory at Next to leading order (NLO) and the exact OPE result.

\begin{tabular}{|c|c|c|c|c|c|c|}
\hline \hline & $\gamma\left(\mathrm{fm}^{-1}\right.$ & $\eta$ & $A_{S}\left(\mathrm{fm}^{-1 / 2}\right)$ & $r_{m}(\mathrm{fm})$ & $Q_{d}\left(\mathrm{fm}^{2}\right)$ & $P_{D}$ \\
\hline OPE- $\eta$ (LO) & Input & 0 & 0.8752 & 1.9423 & 0.1321 & $6 \%$ \\
OPE-pert (NLO) & Input & 0.051 & 0.7373 & 1.6429 & 0.4555 \\
OPE-exact & Input & 0.02633 & $0.8681(1)$ & $1.9351(5)$ & $0.2762(1)$ & $7.88(1) \%$ \\
NijmII & Input & $0.0253(2)$ & $0.8845(8)$ & $1.968(1)$ & $0.271(1)$ & $5.67(4) \%$ \\
Exp. (non-rel.) & 0.231605 & $0.0256(4)$ & $0.8846(9)$ & $1.971(6)$ & $0.2859(3)$ & $5.67(4) \%$ \\
\hline \hline
\end{tabular}

tances. The asymptotic deuteron wave functions for the OPE potential can be written in the form
The first order solution can be evaluated analytically, yielding

$$
\begin{aligned}
& u(r)=e^{-\gamma r}\left[\sum_{k} F_{k}(r) e^{-k m r}\right] \\
& w(r)=\eta e^{-\gamma r}\left(1+\frac{3}{\gamma r}+\frac{3}{(\gamma r)^{2}}\right)\left[\sum_{k} G_{k}(r) e^{-k m r}\right]
\end{aligned}
$$

$$
\begin{aligned}
F_{1}(r) & =\frac{R e^{-m r}\left(m^{2} r^{2}-2(1+r \gamma)-2 m r(1+r \gamma)\right) \eta}{\sqrt{2} r^{3} \gamma^{2}} \\
& +\frac{m^{2} R\left(3 \sqrt{2} m^{2} \eta+\gamma^{2}(4-4 \sqrt{2} \eta)\right) \operatorname{Ei}(-m r)}{12 \gamma^{3}} \\
& +\frac{e^{2 r \gamma} m^{2} R\left(-3 \sqrt{2} m^{2} \eta+4 \gamma^{2}(-1+\sqrt{2} \eta)\right) \operatorname{Ei}(-m r-2 r \gamma)}{12 \gamma} \\
G_{1}(r) & =\frac{m^{2} R\left(-4 \gamma^{2}(\sqrt{2}-2 \eta)+3 m^{2}(\sqrt{2}-\eta)\right) \operatorname{Ei}(-m r)}{12 \gamma^{3} \eta} \\
& +\frac{R e^{-m r}\left(3 m^{3} r(\sqrt{2}-\eta)+m^{2} r \gamma(-3+r \gamma)(\sqrt{2}-\eta)+2 \gamma^{2}(1+m r)(2 \eta+r \gamma(-\sqrt{2}+\eta))\right)}{2 r \gamma^{2}\left(3+3 r \gamma+r^{2} \gamma^{2}\right) \eta} \\
& +\frac{e^{2 r \gamma} m^{2} R\left(3-3 r \gamma+r^{2} \gamma^{2}\right)\left(4 \gamma^{2}(\sqrt{2}-2 \eta)-3 m^{2}(\sqrt{2}-\eta)\right) \operatorname{Ei}(-m r-2 r \gamma)}{12 \gamma^{3}\left(3+3 r \gamma+r^{2} \gamma^{2}\right) \eta}
\end{aligned}
$$

The second order can also be evaluated but the expression is too long to be presented here. In Fig. 11]we present the first order solutions compared to the exact ones. The perturbative solutions of Appendix $\mathrm{A}$ are obtained from the requirement that the $\mathrm{S}$-wave $u$, becomes normalizable when extended down to the origin. This can only hap- pen in the $D / S$ asymptotic ratio, $\eta$ takes the value given by Eq. A16 . This illustrates the fact that perturbation theory can always be applied at long distances but fails at short distances.

Note that here one treats the coupling constant $R$ and the mixing parameter $\eta$ as independent variables.
[1] T. E. O. Ericson and W. Weise, Pions And Nuclei. 1988. Oxford, UK. Clarendon (1988) 479 p. (The International Series of Monographs on Physics, 74).

[2] N. K. Glendenning and G. Kramer, Phys. Rev. 126, 2159
(1962).

[3] S. Klarsfeld, J. Martorell and D. W. L. Sprung, Nucl. Phys. A 352 (1980) 113.

[4] S. Klarsfeld, J. Martorell and D. W. L. Sprung, J. Phys. 


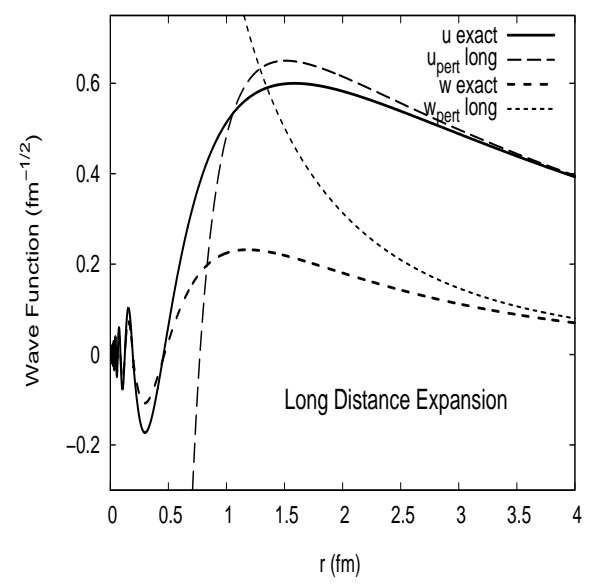

FIG. 11: The long distance expansion for the deuteron wave functions matched. We take $\eta^{\mathrm{OPE}}=0.2633$, and $A_{S}=1$ for the norm of the numerical solutions.

G 10 (1984) 165.

[5] T. E. O. Ericson and M. Rosa-Clot, Phys. Lett. B 110, 193 (1982).

[6] T. E. O. Ericson and M. Rosa-Clot, Nucl. Phys. A 405, 497 (1983).

[7] T. E. O. Ericson and M. Rosa-Clot, Ann. Rev. Nucl. Part. Sci. 35271 (1985).

[8] J. L. Friar, B. F. Gibson and G. L. Payne, Phys. Rev. C 30, 1084 (1984)

[9] J. L. Ballot, A. M. Eiro and M. R. Robilotta, Phys. Rev. C 40, 1459 (1989).

[10] J. L. Ballot and M. R. Robilotta, Phys. Rev. C 45, 986 (1992).

[11] D. W. L. Sprung, W. van Dijk, E. Wang, D. C. Zheng, P. Sarriguren, and J. Martorell Phys. Rev. C 49, 2942 (1994)

[12] S. Weinberg, Phys. Lett. B 251 (1990) 288. S. Weinberg, Nucl. Phys. B 363 (1991) 3.

[13] C. Ordonez, L. Ray and U. van Kolck, Phys. Rev. C 53, 2086 (1996) C. Ordonez, L. Ray and U. van Kolck, Phys. Rev. Lett. 72, 1982 (1994). C. Ordonez and U. van Kolck, Phys. Lett. B 291, 459 (1992).

[14] P. F. Bedaque and U. van Kolck, Ann. Rev. Nucl. Part. Sci. 52, 339 (2002)

[15] M. C. Birse, J. A. McGovern and K. G. Richardson, Phys. Lett. B 464, 169 (1999)

[16] D. B. Kaplan, M. J. Savage and M. B. Wise, Phys. Rev. C 59, 617 (1999)
[17] T. S. Park, K. Kubodera, D. P. Min and M. Rho, Nucl. Phys. A 646, 83 (1999)

[18] T. Frederico, V. S. Timoteo and L. Tomio, Nucl. Phys. A 653, 209 (1999)

[19] E. Epelbaum, W. Gloeckle and U. G. Meissner, Nucl. Phys. A 671, 295 (2000)

[20] D. R. Phillips and T. D. Cohen, Nucl. Phys. A 668 (2000) 45

[21] D. R. Entem and R. Machleidt, Phys. Lett. B 524, 93 (2002)

[22] K. M Case, Phys. Rev. 80, 797 (1950)

[23] W. M. Frank, D. J. Land, and R .M. Spector, Rev. Mod. Phys. 43, 36 (1971).

[24] M. Pavon Valderrama and E. Ruiz Arriola (in preparation)

[25] S. R. Beane, P. F. Bedaque, M. J. Savage and U. van Kolck, Nucl. Phys. A $\mathbf{7 0 0}$ (2002) 377

[26] S. R. Beane, arXiv:hep-ph/0204107

[27] A. Bulgac, G. A. Miller and M. Strikman, Phys. Rev. C 56 (1997) 3307

[28] S. R. Beane and M. J. Savage, Nucl. Phys. A 713, 148 (2003)

[29] E. Epelbaum, U. G. Meissner and W. Gloeckle, Nucl. Phys. A 714, 535 (2003)

[30] S. R. Beane and M. J. Savage, Nucl. Phys. A 717, 91 (2003)

[31] J. J. de Swart, M. C. M. Rentmeester and R. G. E. Timmermans, PiN Newslett. 13, 96 (1997)

[32] T. E. O. Ericson, B. Loiseau and A. W. Thomas, Phys. Rev. C 66 (2002) 014005

[33] V. G. J. Stoks, R. A. M. Klomp, C. P. F. Terheggen and J. J. de Swart, Phys. Rev. C 49, 2950 (1994)

[34] M. Pavon Valderrama and E. Ruiz Arriola, Phys. Lett. B 580, 149 (2004)

[35] M. Pavon Valderrama and E. Ruiz Arriola, Phys. Rev. C 70, 044006 (2004)

[36] D. B. Kaplan, M. J. Savage and M. B. Wise, Nucl. Phys. B 534, 329 (1998)

[37] J. J. de Swart, C. P. F. Terheggen and V. G. J. Stoks, arXiv:nucl-th/9509032

[38] V. G. Stoks, R. A. Kompl, M. C. Rentmeester and J. J. de Swart, Phys. Rev. C 48, 792 (1993).

[39] M. Pavon Valderrama and E. Ruiz Arriola, arXiv:nucl-th/0407113

[40] T. D. Cohen and J. M. Hansen, Phys. Lett. B 440, 233 (1998)

[41] S. Klarsfeld, J. Martorell, J. A. Oteo, M. Nishimura and D. W. L. Sprung, Nucl. Phys. A 456, 373 (1986).

[42] D. W. L. Sprung, Hua Wu and J. Martorell, Phys. Rev. C 42, 863A (1990). 